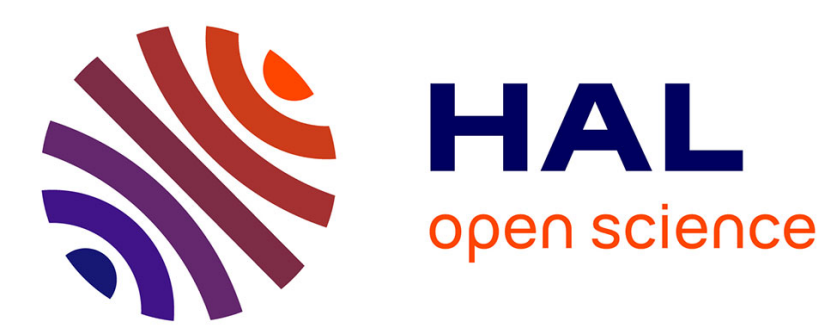

\title{
Modeling and Analysis of Mixed Flow of Cars and Powered Two Wheelers
}

Sosina Gashaw, Paola Goatin, Jérôme Härri

\section{To cite this version:}

Sosina Gashaw, Paola Goatin, Jérôme Härri. Modeling and Analysis of Mixed Flow of Cars and Powered Two Wheelers. Transportation research. Part C, Emerging technologies, 2018, pp.1-44. hal-01708005

\section{HAL Id: hal-01708005 \\ https://hal.inria.fr/hal-01708005}

Submitted on 13 Feb 2018

HAL is a multi-disciplinary open access archive for the deposit and dissemination of scientific research documents, whether they are published or not. The documents may come from teaching and research institutions in France or abroad, or from public or private research centers.
L'archive ouverte pluridisciplinaire $\mathbf{H A L}$, est destinée au dépôt et à la diffusion de documents scientifiques de niveau recherche, publiés ou non, émanant des établissements d'enseignement et de recherche français ou étrangers, des laboratoires publics ou privés. 


\title{
Modeling and Analysis of Mixed Flow of Cars and Powered Two Wheelers
}

\author{
Sosina Gashaw†, Paola Goatin†, Jérôme Härri† \\ $\dagger$ †URECOM, Communication System Department, Sophia Antipolis \\ $\ddagger$ Université Côte d'Azur, Inria, CNRS, LJAD, France
}

\begin{abstract}
In modern cities, a rapid increase of motorcycles and other types of Powered Two-Wheelers (PTWs) is observed as an answer to long commuting in traffic jams and complex urban navigation. Such increasing penetration rate of PTWs creates mixed traffic flow conditions with unique characteristics that are not well understood at present. Our objective is to develop an analytical traffic flow model that reflects the mutual impacts of PTWs and Cars. Unlike cars, PTWs filter between cars, have unique dynamics, and do not respect lane discipline, therefore requiring a different modeling approach than traditional "Passenger Car Equivalent" or "Follow the Leader". Instead, this work follows an approach that models the flow of PTWs similarly to a fluid in a porous medium, where PTWs "percolate" between cars depending on the gap between them.

Our contributions are as follows: (I) a characterization of the distribution of the spacing between vehicles by the densities of PTWs and cars; (II) a definition of the equilibrium speed of each class as a function of the densities of PTWs and cars; (III) a mathematical analysis of the model's properties (IV) an impact analysis of the gradual penetration of PTWs on cars and on heterogeneous traffic flow characteristics.
\end{abstract}

Keywords: Multiclass traffic flow model, Powered two wheelers, Porous flow, Traffic impacts analysis

\section{Introduction}

While a car is seen as a social achievement in most of the eastern coun- 
of Powered Two-Wheeler (PTW) to mitigate their perceived impact of traffic congestion (e.g. reduced travel time). In some cities, electrical scooter sharing initiatives are also proposed for drivers to switch transportation modes when reaching city centers. The significantly growing use of PTWs calls for new technologies to integrate PTWs safely and efficiently with other road users. Thus far, the focus is mainly on solving the safety issues of PTWs. However, the other aspect, i.e. traffic flow efficiency, has not been addressed sufficiently. Emerging intelligent transport system (ITS) technologies would play an important role in improving traffic mobility of PTWs as well as other users. This would be achieved by reducing the influence of PTWs on other road users, for example at intersections. Additionally, the opportunity provided by PTWs could be exploited effectively by introducing a cooperation between PTWs and other interacting vehicles. Other 'PTWs aware' technologies could also contribute to promote PTWs use, which in turn minimize congestion.

Yet, PTWs create traffic flow effects Yet, PTWs create traffic flow effects (e.g. car flow reduction in presence of PTWs, PTW filtering between and up car streams, etc...) that are difficult to understand with the currently available models. Without such understanding, it is difficult to evaluate or develop innovative transportation solutions with or for PTWs, such as adapting traffic lights management to mixed traffic, safety-related PTW applications such as collision/approach warnings, or multi-modal initiatives.

The interaction between PTWs and cars creates mixed traffic flow situations, for which state-of-art models are not adapted. Multi-class flow modeling arises as an effort to characterize such mixed traffic flow situations, which may be characterized roughly in two domains: Mixed "driver" characteristics (Daganzo, 2002) or mixed "vehicle" characteristics. In this work, we focus on the latter case, where a classification among the vehicle classes is made on lane specific patterns, vehicles physical and dynamical features, and where each vehicle in a class possesses identical characteristics (Logghe and Immers, 2008).

In a microscopic approach, the heterogeneity of driver and vehicle characteristics is modeled by defining different behavioral rules and parameters such as longitudinal and lateral movement rule (Pandey et al., ????), speed choice, headway (Lenorzer et al., 2015), reaction time, etc. The parameters and driver behaviors are described differently depending on the interacting vehicle classes (SHIOMI et al., 2012). Space discretization methods are also introduced to accommodate lateral movement within a lane and the variation 
in vehicle size (Chen et al., 2013; Mathew et al., 2013).

Multi-class traffic flows are usually evaluated following a metric called "Passenger Car Equivalent" (PCE), which reports the impact of a given class of traffic on traffic flow variables. With PCE a heterogeneous traffic flow is converted to a hypothetical homogeneous flow by representing the influence of each vehicle in terms of the equivalent number of passengers per car. PCE value for vehicles varies with the traffic conditions (Praveen and Arasan, 2013) and the value should be selected depending on traffic speed, vehicles' size, headway and other traffic variables (Adnan, 2014). However, only few models Van Lint et al. (2008) define traffic state dependent PCE value.

Numerous multi-class models are stemmed from the desire to characterize mixed flows of cars and trucks. For instance, the model in (Zhang and Jin, 2002) formulates a mixed flow of passenger cars and trucks based on their free flow speed difference. A two-class flow model proposed in (Chanut and Buisson, 2003) differentiates vehicles according to their length and speed. Furthermore, heterogeneity among vehicles is modeled relating to maximal speed, length and minimum headway of vehicles in (Van Lint et al., 2008). Despite providing a separate representation for each vehicle classes, in all these models (Chanut and Buisson, 2003; Van Lint et al., 2008; Zhang et al., 2006) vehicle classes have identical critical and jam density parameters, but the parameters are scaled according to the actual traffic state. The multiclass model in (Wong and Wong, 2002) extends LWR model for heterogeneous drivers by distinguishing the vehicle classes by the choice of the speed. The assumption is that drivers respond in a different way to the same traffic density. Correspondingly, the work in (Benzoni-Gavage and Colombo, 2003) presents a mixed flow for several populations of vehicles, where the vehicle classes are differentiated by the maximal speed, and the equilibrium speed is expressed as a function of total occupied space. The model in (Fan and Work, 2015) uses a similar approach, yet integrating a specific maximum occupied space for each vehicle class.

Mixed flows consisting of PTWs yet exhibit distinctive features from the assumption taken in the previously described multi-class models, making them look more like disordered flows without any lane rule. Their narrow width indeed grants PTWs flexibility to share lanes with other vehicles or filter through slow moving or stationary traffic, requiring traffic stream attributes to be defined differently from traffic following lane rules (Mallikarjuna and Rao, 2006). Accordingly, Nair et al. (2011) proposed to model 
PTWs as a fluid passing through a porous medium. The speed-density relationship is presented in terms of pore size distributions, which Nair et al. obtained through exhaustive empirical simulations. This approach is computational very expensive and hardly reproducible, as it requires a different set up for each scenario being considered. On a later work from the same authors (Nair et al., 2012), the pore size distribution is assumed to follow an exponential distribution. Yet, the distribution parameter $\lambda$ is defined wrongly, i.e. the mean pore size increases with increasing of vehicle class densities. Furthermore, the mean pore size is not described uniquely for given vehicle-classes densities.

Therefore, this paper focuses specifically on a more realistic modeling of the pore size distribution, which is critical to mixed flow models based on a porous medium strategy. Our first contribution provides an enhanced mixed flow modeling, where we: (i) provide a closed form analytical expression for the pore size distribution and the statistical parameters of the pore size distribution (mean, variance and standard deviation) for generic traffic flow consisting of cars and PTWs; (ii) propose a fundamental relation described as a function of the density of each vehicle class. The fundamental diagram and the parameters for the fundamental diagram are defined uniquely for each class, and are also adapted to the traffic condition; (iii) Provide a mathematical analysis of the model's properties (iv) apply a consistent discretization method for the approximation of the conservation equations. Our second contribution evaluates the impact of our enhanced model to traffic flow characteristics, where we: (i) evaluate the impact of the maximum road capacity; (ii) formulate mixed flow travel time; (iii) analyze traffic light clearance time, and this considering a gradual increase of PTWs.

The proposed model contributes as an enabler for 'PTW aware' emerging technologies and traffic regulations. For example, a variety of traffic control strategies require traffic flow models to predict the traffic state and make an appropriate control decision. Employing our model in such system opens a door to the inclusion of PTWs in traffic control. On the other hand, the model can be used as a framework to assess the optimality of the existing control schemes, including information collection and computation methods. Moreover, the model could help traffic regulator to determine collective and class-specific optima and to induce a vehicle class specific flow adjustment. In this way, new traffic regulations adapted to PTWs can be introduced, which in turn promotes the use of PTWs. Additionally, our model could be applied to design a smart two-wheeler navigation system which is well 
aware of PTWs' capability to move through congested car traffic and provides a route plan accordingly. The model could also contribute in the proper integration of PTWs into multi-modal transport planning. In general, the model plays a role in enabling 'PTW aware' traffic efficiency related applications/technologies.

\section{Model description}

One of the most used macroscopic models is the first order model developed by Lighthill, Whitham and Richards (Lighthill and Whitham, 1955; Richards, 1956). In the LWR model, traffic flow is assumed to be analogous to one-directional fluid motion, where macroscopic traffic state variables are described as a function of space and time. Mass conservation law and the fundamental relationship of macroscopic state variables, namely, speed, density, and flow are the basic elements for LWR formulation. The conservation law says that with no entering or leaving vehicles the number of vehicles between any two points is conserved. Thus, the first order PDE equation based on the conservation law takes the form

$$
\frac{\partial \rho(x, t)}{\partial t}+\frac{\partial q(x, t)}{\partial x}=0
$$

where $\rho(x, t), q(x, t)$ are, respectively, the density and the flow of cars at position $x$ and time $t$. Flow $q(x, t)$ is expressed as function of the traffic state variables:

$$
q(x, t)=\rho(x, t) v(x, t)
$$

The speed $v(x, t)$ depends on the density and a unique speed value corresponds to a specific traffic density, i.e.

$$
v(x, t)=V(\rho(x, t)) .
$$

In the original LWR model, all vehicles in a traffic stream are considered to exhibit similar characteristics. Therefore, no classification is made between vehicle classes. Multi-class extensions of the LWR model emerge to accommodate the heterogeneity in many aspects of road users. In multi-class modeling, vehicles with identical characteristics are grouped into a class and a conservation law applies to each class. For two vehicle classes the conservation equation is written as

$$
\frac{\partial \rho_{i}(x, t)}{\partial t}+\frac{\partial q_{i}(x, t)}{\partial x}=0, \quad i=1,2,
$$


where $\rho_{i}$ and $q_{i}$ denote density and flow of class i, respectively. Class specific flow, speed and density are related by the equations

$$
q_{i}(x, t)=\rho_{i}(x, t) v_{i}(x, t), \quad i=1,2 .
$$

The equilibrium speed $v_{i}$ for the individual vehicle class $i$ is a function of the densities of both classes and satisfies the following conditions:

$$
v_{i}=V_{i}\left(\rho_{1}, \rho_{2}\right), \quad \partial_{1} V_{i}\left(\rho_{1}, \rho_{2}\right) \leq 0, \partial_{2} V_{i}\left(\rho_{1}, \rho_{2}\right) \leq 0,
$$

where $\partial_{1} V_{i}\left(\rho_{1}, \rho_{2}\right)$ and $\partial_{2} V_{i}\left(\rho_{1}, \rho_{2}\right)$ denote $\frac{\partial V_{i}\left(\rho_{1}, \rho_{2}\right)}{\partial \rho_{1}}$ and $\frac{\partial V_{i}\left(\rho_{1}, \rho_{2}\right)}{\partial \rho_{2}}$, respectively. The interaction among vehicle classes is captured through the equilibrium speed. Moreover, the equilibrium speed is uniquely defined for all points of the space

$$
S=\left\{\left(\rho_{1}, \rho_{2}\right): \rho_{1}<=\rho_{1}^{j a m}\left(\rho_{2}\right), \rho_{2}<=\rho_{2}^{j a m}\left(\rho_{1}\right)\right\}
$$

where $\rho_{1}^{\text {jam }}\left(\rho_{2}\right)$ and $\rho_{2}^{j a m}\left(\rho_{1}\right)$ are the jam densities of vehicle class 1 and 2 , respectively. In this model, we adopt the speed function proposed in (Nair et al., 2011). This speed-density relationship is derived based on the assumption that the flow of vehicles is dictated by available free spaces along the way, and it is written as

$$
\left.v_{i}=v_{i}^{f}\left(1-\int_{0}^{r_{i}^{c}} f\left(l\left(\rho_{1}, \rho_{2}\right)\right)\right) \mathrm{d} l\right),
$$

where $f\left(l\left(\rho_{1}, \rho_{2}\right)\right), v^{f}$ and $r^{c}$ are, respectively, the probability density function (PDF) of the inter-vehicle spacing (pore), the free speed and the minimum traversable inter-vehicle space (critical pore size) of class $i$. As such, by relating the speed to the inter-vehicle spacing lane sharing, filtering and creeping behaviors of PTWs can be captured, rendering it more suitable for our purpose than any other multi-class speed functions. However, in (Nair et al., 2011) a closed form expression for the PDF of inter-vehicle spacing (pore) is missing. The same author later proposes exponential distribution (Nair et al., 2012) with intensity $\lambda$ to characterize the inter-vehicle spacing, where $\lambda$ is given as:

$$
\lambda=\left(l_{\text {max }}-l_{\text {min }}\right)\left(1-\sum_{i=1}^{2} a_{i} \rho_{i}\right)+l_{\text {min }} .
$$

This definition of the distribution parameter $\lambda$ produces an incorrect result, i.e. the speed increases with increasing of vehicle class densities. Furthermore, it does not describe the equilibrium speed uniquely for a given class 


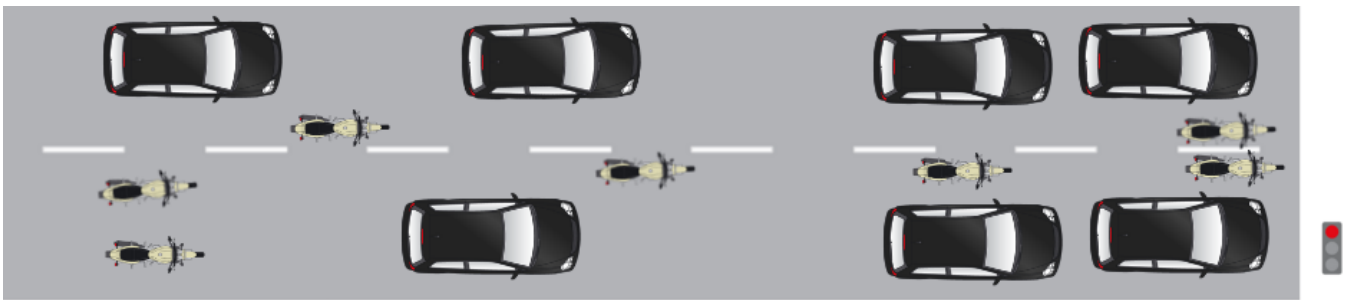

Figure 1: Heterogeneous traffic flow for PTWs and cars.

densities ( the requirement defined in equation (6)). On the other hand, the exponential assumption is taken based on the longitudinal headway distribution. In order to fill this gap, we develop an analytical expression for the inter-vehicle spacing distribution based on simulation results. Further, we introduce an approximation method in order to determine the distribution parameters.

\subsection{Vehicle spacing distribution}

Vehicle-spacing distribution, which was referred as pore space distribution, was first used to describe the speed-density relationship in the paper by Nair et al. (2011), yet the distribution was not known. Here, we propose Poisson point process and Delaunay triangulation based method for the derivation of vehicle spacing distribution.

For the sake of simplicity, we take the following assumptions: cars and PWTs have a circular shape and they are distributed in the domain uniformly and independently according to Poisson point process with intensity $\lambda$, where $\lambda$ is the number of vehicles per unit area. Although limited to non-dense traffic, the study done using real data in (Jiang et al., 2016) supports the Poisson point process assumption for the spatial distribution of vehicles on the road. The circular shape of vehicles that is introduced for simplification does not change the distribution of the inter-vehicle spacing qualitatively. Furthermore, Delaunay triangulation is used to define the spacing between vehicles on the assumption that Delaunay triangle edge length represents the size of the spacing.

Given the density of each vehicle classes, vehicles are placed uniformly and independently without overlapping in a two-dimensional finite space with intensity $\lambda=\rho_{1}+\rho_{2}$. Here, $\rho_{1}$ and $\rho_{2}$ represent PTWs' and cars' areal density, i.e. vehicles per unit area, respectively. The Delaunay triangulation is constructed over the center of vehicles (Figure 2(a)) and the triangles edge 


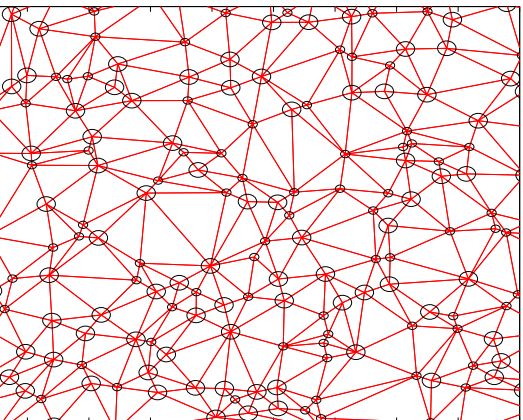

(a) Delaunay triangulation over vehicles, one example scenario. density function (Figure 2(b)).

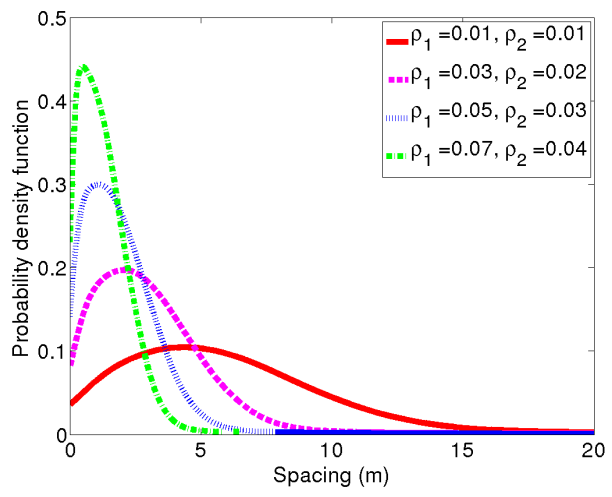

(b) Probability density function for different traffic compositions.

Figure 2: Vehicles spacing distribution, where $\rho_{1}$ and $\rho_{2}$ represent, respectively, PTWs

length data from multiple simulation runs is used to estimate the probability

\section{and cars density}

In (Miles, 1970) it is indicated that for a Delaunay triangulation performed on homogeneous planar Poisson point with intensity $\lambda$ the mean value of the length of Delaunay triangle edge, and the square of the length are given by $E\left(l_{p}\right)=\frac{32}{9 \pi \sqrt{\lambda}}$ and $E\left(l_{p}^{2}\right)=\frac{5}{\pi \lambda}$, respectively. We then convert these formulations to our problem where we have circles, instead of points. When the points are replaced by circles (small circles for PTWs and large circles for cars), edge length measured for points is reduced by the sum of the radius of the circles the two end points of the edge. For instance, an edge connecting PTWs and cars is reduced by $R_{1}+R_{2}$, where $R_{1}$ and $R_{2}$ are the radius of a circle representing the PTW and the car respectively. 


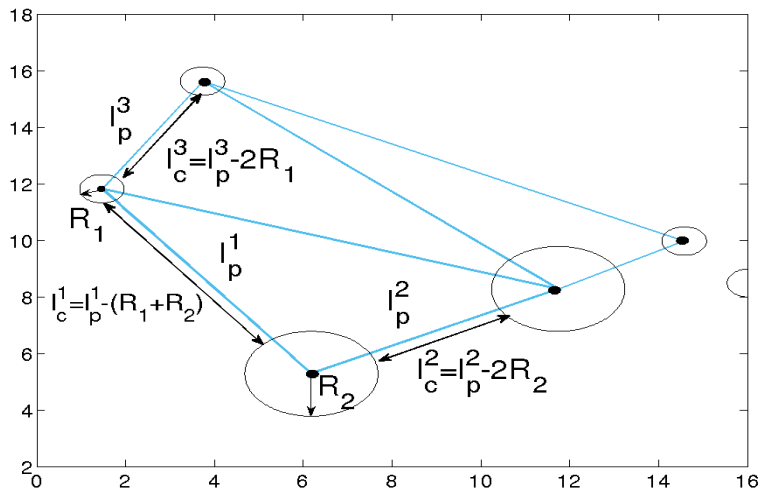

Figure 3: Delaunay triangle edges length for circles.

In accordance with the mean length of the delaunay traingle edge over points, we define for circles as (Figure 3):

$$
E\left[l_{c}\right]=E\left[l_{p}\right]-2\left(R_{1} p_{1}+R_{2} p_{2}\right),
$$

where $p_{1}$ is probability for an edge to touch PTWs and $p_{2}$ for cars. This probability is expressed in the form $p_{i}=\frac{\rho_{i}}{\rho_{1}+\rho_{2}}$, therefore we get

$$
E\left(l_{c}\right)=\mu=\frac{32}{9 \pi \sqrt{\rho_{1}+\rho_{2}}}-\frac{2\left(R_{1} \rho_{1}+R_{2} \rho_{2}\right)}{\rho_{1}+\rho_{2}} .
$$

Standard deviation and variance are the same for the case of points $\left(\sigma_{p}^{2}\right)$ and circles $\left(\sigma_{c}^{2}\right)$, thus

$$
\sigma_{p}^{2}=E\left(L_{p}^{2}\right)-E\left(L_{p}\right)^{2} \approx \frac{3}{\pi^{2} \lambda}, \quad \sigma_{c}^{2}=\frac{3}{\pi^{2}\left(\rho_{1}+\rho_{2}\right)} .
$$

The above equations provide the parameters for the distribution function of inter vehicle-spacing, we then identify the best fitting theoretical distribution. To determine a theoretical probability density function (PDF) that best fits the observed PDF, we use MATLAB's curve fitting tool, and the goodness of the fit is measured by R-square, sum of squared errors (SSE) and root mean square error (RMSE) values. We consider left-truncated normal, log-normal 
and exponential as candidate distributions to characterize vehicle-spacing. The distributions are chosen based on qualitatively observed similarity on the shape of PDF curve. We also include the exponential distribution, which is recommended in (Nair et al., 2012). The comparison between the three selected theoretical distribution functions is shown in Figure 4. Based on the goodness of the fit results, see Table 1, left-truncated normal (LT-Normal) distribution conforms better to the estimated PDF than the other distributions. Besides, it can be noted that the negative exponential assumption taken in (Nair et al., 2012) is not fitting well.

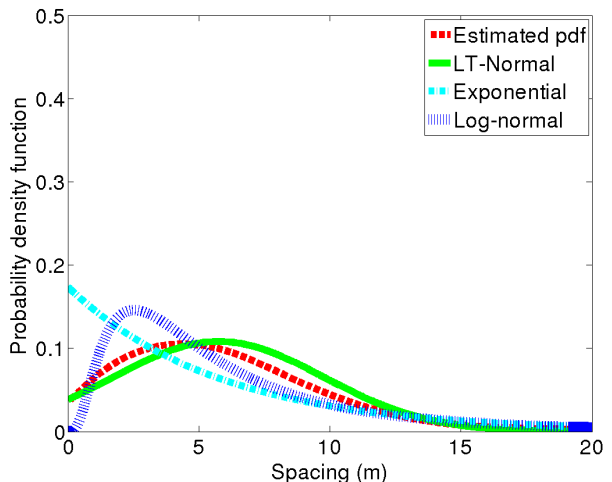

(a) Fitting plot for $\rho_{1}=0.01, \rho_{2}=0.01, v / m^{2}$

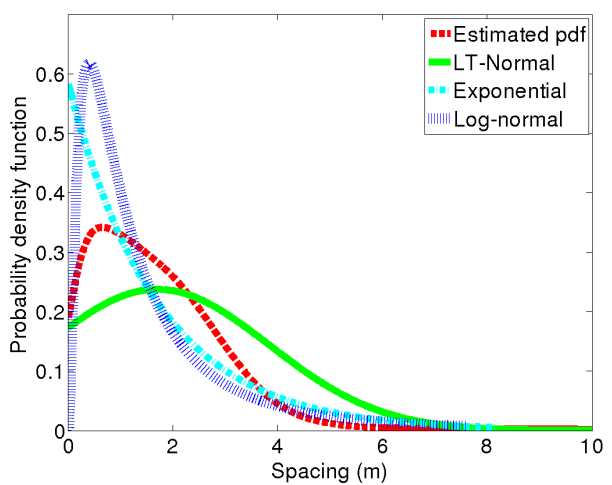

(c) Fitting plot for

$$
\rho_{1}=0.02, \rho_{2}=0.05, v / m^{2}
$$

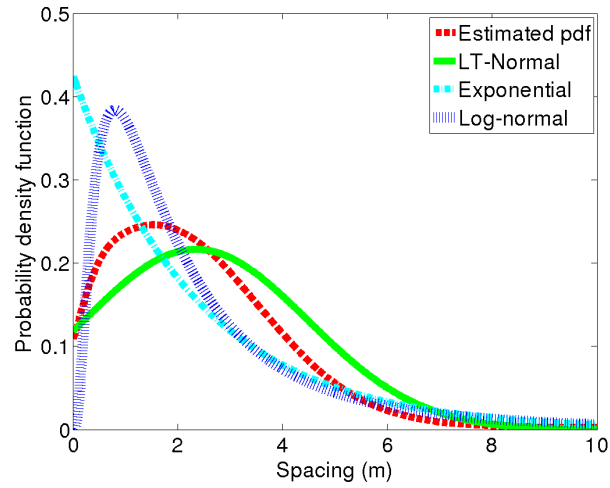

(b) Fitting plot for $\rho_{1}=0.05, \rho_{2}=0.02, v / m^{2}$

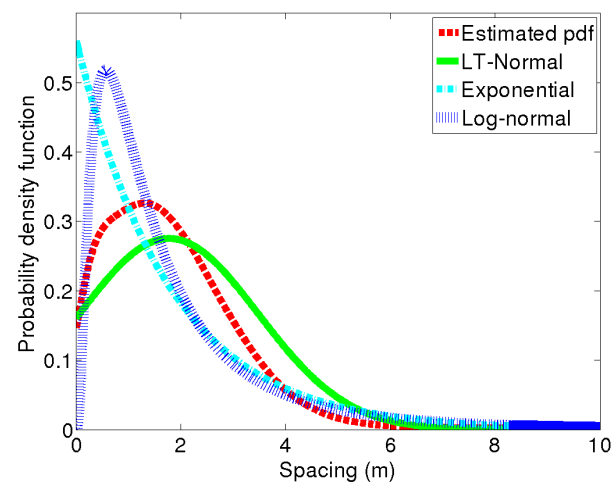

(d) Fitting plot for $\rho_{1}=0.1, \rho_{2}=0.01, v / m^{2}$

Figure 4: Comparison of estimated probability distribution function and fitting theoretical distributions for different vehicles composition 


\begin{tabular}{lccc|ccc} 
& SSE & R-square & RMSE & SSE & R-square & RMSE \\
\hline \hline & \multicolumn{3}{c}{$\rho_{1}=0.01, \rho_{2}=0.01$} & \multicolumn{3}{c}{$\rho_{1}=0.05, \rho_{2}=0.02$} \\
LT-normal & 0.24 & 0.955 & 0.0069 & 0.97 & 0.938 & 0.0139 \\
Log-normal & 0.809 & 0.851 & 0.0127 & 2.67 & 0.831 & 0.023 \\
Exponential & 2.17 & 0.602 & 0.0208 & 4.05 & 0.744 & 0.028 \\
\hline \multicolumn{4}{c}{$\rho_{1}=0.02, \rho_{2}=0.05$} & \multicolumn{2}{c}{$\rho_{1}=0.1, \rho_{2}=0.01$} \\
LT-normal & 3.21 & 0.853 & 0.025 & 1.46 & 0.993 & 0.017 \\
Log-normal & 5.51 & 0.748 & 0.033 & 4.07 & 0.813 & 0.028 \\
Exponential & 3.93 & 0.82 & 0.028 & 5.39 & 0.753 & 0.032 \\
\hline
\end{tabular}

Table 1: Goodness of the fit measures obtained from the fitting experiments for different theoretical distributions.

We added minimum distance rejection criteria (minimum allowable distance) to Poisson point process distribution so that vehicles do not overlap, resulting in change of inter vehicle spacing distribution property ( E.g. the average, variance...of the distribution ). Due to this, we observed that the road width and ratio of vehicle classes have an influence on the PDF. The effect of the size of the area is pronounced when $L>>W$, where $L$ and $W$ denote length and width of the area (see Figure 5). The significance of the variation also depends on the ratio of the two densities. Yet, left-truncated normal distribution remains the best fit and gives a good approximation in most of the situation.

Therefore, we assume that the spacing distribution follows the left-truncated normal distribution, having the form

$$
f_{p T N}(l)=\left\{\begin{array}{ll}
0 & l<0 \\
\frac{f_{p}(l)}{\int_{0}^{\infty} f_{p}(l)} & l \geq 0
\end{array} \quad \text { where } f_{p}=\frac{1}{\sqrt{2 \pi} \sigma} \exp \frac{-(x-\mu)^{2}}{2 \sigma^{2}} .\right.
$$

\subsection{Speed-density relationship}

Using the PDF function in equation (8), the speed-density relationship in equation (7) is re-written as

$$
v_{i}=v_{i}^{f}\left(1-\int_{0}^{r_{i}^{c}} f_{p T N}(l) \mathrm{d} l\right),
$$

where $v_{i}^{f}$ and $r_{i}^{c}$ represent the free flow speed and the critical pore size, respectively, of class i. The critical pore size depends on the traffic situation 


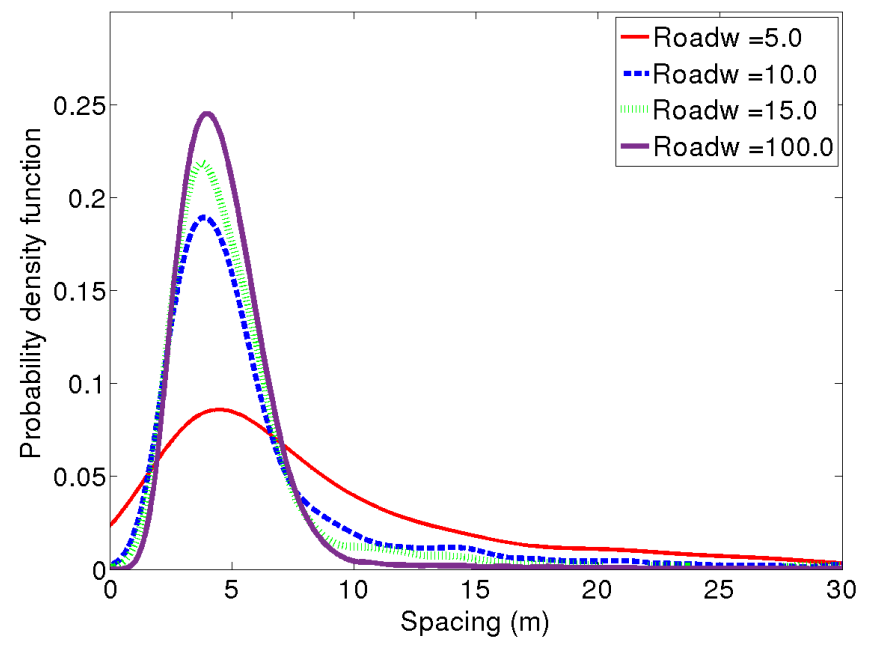

Figure 5: Example $\rho_{1}=\rho_{2}=0.03 \mathrm{veh} / \mathrm{m}^{2}$ : PDF of the inter-vehicle distance on a road with dimension $L=100$ and $W$ ranging from $5 m-100 m$

and the interacting vehicle class (Ambarwati et al., 2014). The critical pore size accepted by vehicles when travelling at higher speed is larger than the critical pore size at lower speeds. To reproduce the critical pore size - speed proportionality (Minh et al., 2012), for example, we can formulate the critical pore size as:

$$
r_{c}=r_{c}^{m i n}+r *\left(1-\left(\rho_{1} * A_{1}+\rho_{2} * A_{2}\right)\right),
$$

where $\rho_{1}, A_{1}, \rho_{2}, A_{2}, r_{c}^{\min }$ and $\mathrm{r}$ denote density of PTW, area of PTW, density of car, area of car, the minimum critical pore size, and the difference between the maximum and the minimum critical pore size, respectively. As such, the critical pore size increases with increasing speed or with decreasing vehicle class densities, which is in agreement with the gap acceptance theory. To evaluate the impact of the critical pore on the speed function, we compare the result for a constant critical pore size and a critical pore size scaled according to the actual traffic. As depicted in Figure 6, the critical pore size doesn't change the qualitative behavior of our fundamental diagram. Since the critical pore size does not have any qualitative implication, for simplicity we use a constant value. The limitation of equation (9) is that, because of the property of normal distribution function, the speed becomes zero only at infinite density, as for the speed function used in (Nair et al., 2011). In attempt to overcome this infinite jam density, we have distinguished the jam 


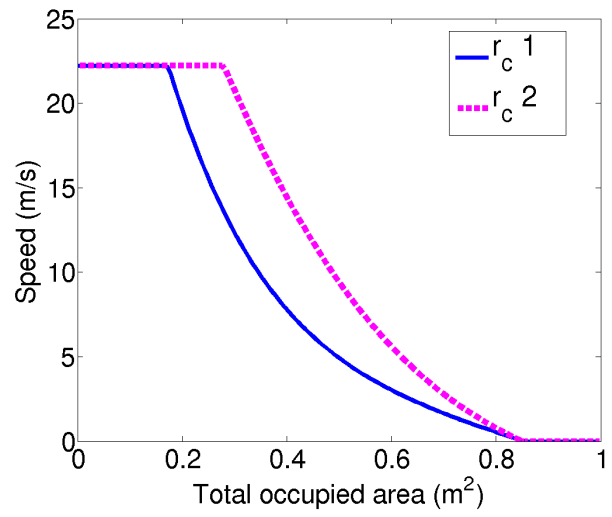

Figure 6: Speed vs total occupied area for constant critical pore size $\left(r_{c}^{2}=3 m\right)$ and a variable critical pore size $\left(r_{c}^{1}\right)$ with the following parameters $r_{c}^{\min }=3 \mathrm{~m}$ and $r=2 \mathrm{~m}$.

area occupancy for the two classes, and the speed values are normalized to zero at the jam area occupancy. Beside the consideration of vehicles size, we selected the jam area occupancies for the two classes in such a way to allow filtering of PTWs through completely stopped cars traffic (Fan and Work, 2015). We distinguish the maximum total occupied area, which is the extreme total occupied areas corresponding to the null speed of a vehicle class, for the two classes in such a way that

$$
V_{2}\left(A_{\max }^{2}\right)=0, V_{1}\left(A_{\max }^{2}\right)>0, V_{2}\left(A_{\max }^{1}\right)=V_{1}\left(A_{\max }^{1}\right)=0, A_{\max }^{2}<A_{\max }^{1}
$$

where $V_{2}, V_{1}, A_{\max }^{2}$ and $A_{\max }^{1}$ represent the speed of cars, the speed of PTWs, the maximum total occupied area of cars and the maximum total occupied area of PTWs, respectively. Accordingly, when the total area occupied by vehicles equals $A_{\max }^{2}$, the cars completely stop while the average speed of PTWs is greater than zero. Due to this, PTWs can move through jammed car until the total area occupied by vehicles reaches to $A_{\text {max }}^{1}$. On the grounds of the relation in eqn. (10) and some realistic conditions, we approximate the jam area occupancy, i.e. $\rho_{1} A_{1}+\rho_{2} A_{2}$, to 1 for PTWs and to 0.85 for cars, where $\rho, A$ stand for density $(v e h / m 2)$ and projected area of vehicles $\left(m^{2}\right)$, respectively. 


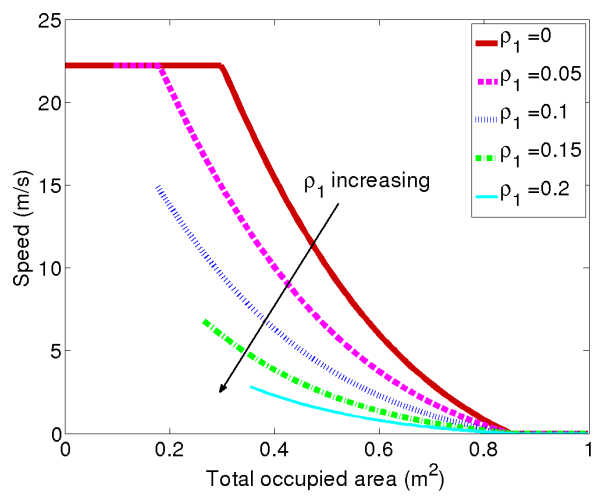

(a) Car speed at different density of PTWs.

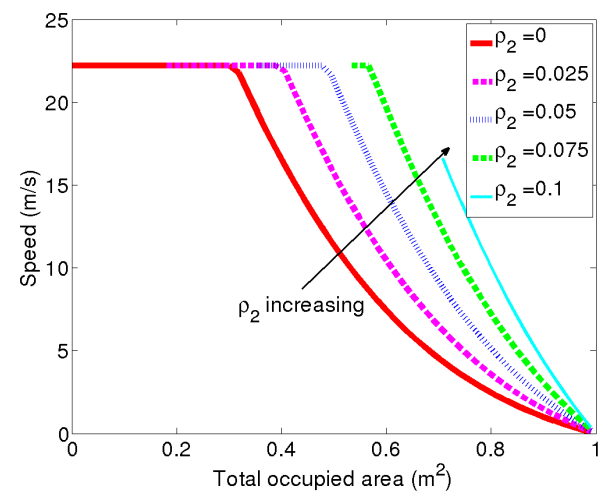

(b) PTWs speed at different cars density values.

Figure 7: Speed Vs total occupied area $\left(\sum \rho_{1} A_{1}+\sum \rho_{2} A_{2}\right)$, where $\rho_{1} A_{1}$ and $\rho_{2} A_{2}$ are area projected on the road by PTW and car, respectively.

Further modification is applied to the speed function in order to comply with triangular fundamental diagram theory, that is presence of two regimes, specifically, congestion and free flow regime (Newell, 1993). In free flow there is no significant drop of average speed with the increase of density. However, beyond some critical density value, the average speed of vehicles decreases with density increase. Therefore, we adjust the speed functions to:

$$
v_{1}=\min \left\{v_{1}^{f}, C_{v} v_{1}^{f}\left(1-\frac{1}{N_{1}} \int_{0}^{r_{1}^{c}} f_{p T N}(l) \mathrm{d} l\right)\right\},
$$

$$
v_{2}=\min \left\{v_{c}^{f}, C_{v} v_{2}^{f}\left(1-\frac{1}{N_{2}} \int_{0}^{r_{2}^{c}} f_{p T N}(l) \mathrm{d} l\right)\right\},
$$

where $N_{i}$ is a speed normalization factor and $C_{v}$ is a scaling factor so that $v_{i}$ equals the free flow speed at critical density in the presence of traffic of vehicle class $i$ only. After all the modifications, the speed-density relation look as shown in Figure 7. Different from the existing models which describe traffic composition in terms of total area/space occupancy (Nair et al., 2012) (Fan and Work, 2015)(Benzoni-Gavage and Colombo, 2003), one of the key characteristics of our speed model is that it captures well the variation in traffic composition as the speed is expressed as a function of the density of each vehicle class. Specifically, for a given area occupancy, depending on the proportion of one class of vehicles the speed value varies. For instance, for a 
given area occupancy, the higher the percentage of PTWs the higher becomes the number of vehicles and the average pore size shrinks. In turn, the speed value decreases. The general properties of our speed model are summarized as follows:

1. A unique speed value is associated with a given total density and traffic composition.

2. In free flow, vehicles move at constant (maximal) speed.

3. In congestion, speed decreases with increase of density.

4. Speed depends on the densities of the two vehicle classes and their proportion.

5. For the same occupancy area (total area occupied by vehicles) the more the share of PTWs is the lower becomes the speed, which is the main property missed by multi-class models that define the speed function in terms of area occupancy.

6. Each class has a different fundamental relation

7. Each class has a distinctive critical and jam densities parameters.

None of the models known to us satisfies all the aforementioned properties, although there are models that satisfy a few of them. Property (3), (4) and (6) are common to most of multi-class LWR models. Nonetheless, models that describe speed as a function of total occupied space (Benzoni-Gavage and Colombo, 2003; Fan and Work, 2015; Chanut and Buisson, 2003) do not satisfy property (1). While (Van Lint et al., 2008) satisfies property (1) and (Fan and Work, 2015) satisfies property (7), property (5) is unique to our model.

\subsection{Model Analysis}

To describe the solution of the system equations (3)-(5) in terms of wave motion, the jacobian matrix $D q$ of $q=\left(q_{1}, q_{2}\right)$ should be diagonalizable with real eigenvalues, in another word the system has to be hyperbolic. We can prove the hyperbolicity by showing that the system is symmetrizable, i.e. there exists a positive-definite matrix $S$ such that $S D q$ is symmetric, see (Benzoni-Gavage and Colombo, 2003).

Re-writing the system in the form:

$$
\frac{\partial \rho}{\partial t}+D q(\rho) \frac{\partial \rho}{\partial x}=0,
$$


where

$$
\rho=\left[\begin{array}{l}
\rho_{1} \\
\rho_{2}
\end{array}\right] \quad \text { and } \quad q(\rho)=\left[\begin{array}{l}
\rho_{1} v_{1}(\rho) \\
\rho_{2} v_{2}(\rho)
\end{array}\right]
$$

the Jacobian matrix of $q(\rho)$ is given by:

$$
D q(\rho)=\left[\begin{array}{cc}
\frac{\partial\left(\rho_{1} v_{1}\right)}{\partial \rho_{1}} & \frac{\partial\left(\rho_{1} v_{1}\right)}{\partial \rho_{2}} \\
\frac{\partial\left(\rho_{2} v_{2}\right)}{\partial \rho_{2}} & \frac{\partial\left(\rho_{2} v_{2}\right)}{\partial \rho_{1}}
\end{array}\right]=\left[\begin{array}{cc}
\rho_{1} \partial_{1}\left(v_{1}\right)+v_{1} & \rho_{1} \partial_{2}\left(v_{1}\right) \\
\rho_{2} \partial_{1}\left(v_{2}\right) & \rho_{2} \partial_{2}\left(v_{2}\right)+v_{2}
\end{array}\right]
$$

For $\rho_{1}>0, \rho_{2}>0$,

$$
S=\left[\begin{array}{cc}
\frac{1}{\rho_{1} \partial_{2}\left(v_{1}\right)} & 0 \\
0 & \frac{1}{\rho_{2} \partial_{1}\left(v_{2}\right)}
\end{array}\right]
$$

is a symmetrizer of $D q$, thus the system satisfies the hyperbolicity condition.

The eigenvalues of the Jacobian representing information propagation (characteristic) speed are given by:

$$
\lambda_{1,2}=\frac{1}{2}\left[\alpha_{1}+\alpha_{2} \pm \sqrt{\left(\alpha_{1}-\alpha_{2}\right)^{2}+4 \rho_{1} \rho_{2} \partial_{2}\left(v_{1}\right) \partial_{1}\left(v_{2}\right)}\right]
$$

where

$$
\alpha_{1}=\rho_{1} \partial_{1}\left(v_{1}\right)+v_{1}, \quad \alpha_{2}=\rho_{2} \partial_{2}\left(v_{2}\right)+v_{2} .
$$

Following (Benzoni-Gavage and Colombo, 2003, Proposition 3.1) it is possible to show that

$$
\lambda_{1} \leq \min \left\{\alpha_{1}, \alpha_{2}\right\} \leq \min \left\{v_{1}, v_{2}\right\} \text { and } \min \left\{v_{1}, v_{2}\right\} \leq \lambda_{2} \leq \max \left\{v_{1}, v_{2}\right\},
$$

where, we have taken $\lambda_{1} \leq \lambda_{2}$. The proof in (Benzoni-Gavage and Colombo, 2003) assumes that $V_{1}>V_{2}$ to exclude the degenerate case, when $V 1=$ $V 2$. However, Zhang et al. (Zhang et al., 2006) also studied the properties of a similar model as in (Benzoni-Gavage and Colombo, 2003), but here for a generic speed function which is expressed as a function of total density, i.e. $v_{i}=v_{i}(\rho)$, where $\rho=\sum_{i} \rho_{i}$. Accordingly, it is proved that for $v_{1}<v_{2}<v_{3} \ldots<v_{m}$, the eigenvalues are bounded such that $\lambda_{1}<v_{1}<\lambda_{2}<v_{2}<\lambda_{3}<\ldots v_{m}-1<\lambda_{m}<v_{m}$ (refer (Zhang et al., 2006, Theorem 3.1, Lemma 2.2, Lemma 2.3)). Due to the complexity of the dependency of the speed function on vehicle class densities, we could not follow a similar analytical approach. Nonetheless, we have checked the validity 


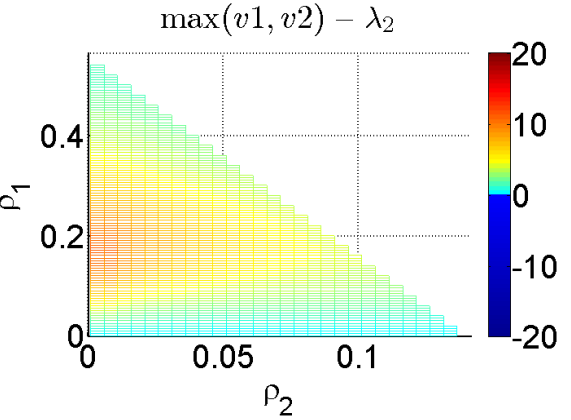

(a) $\max \left(V_{1}, V_{2}\right)-\lambda_{2}$

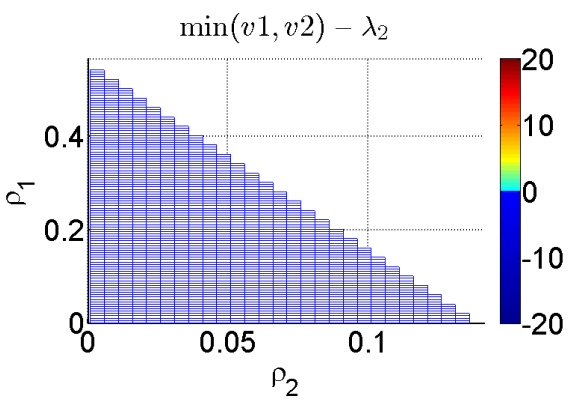

(b) $\min \left(V_{1}, V_{2}\right)-\lambda_{2}$

Figure 8: Evaluation of the maximum characteristics speed over a point in $S=\left\{\rho_{1}, \rho_{2}\right\}$, Here $V_{1}=22 \mathrm{~m} / \mathrm{s}$ and $V_{2}=27 \mathrm{~m} / \mathrm{s}$ 


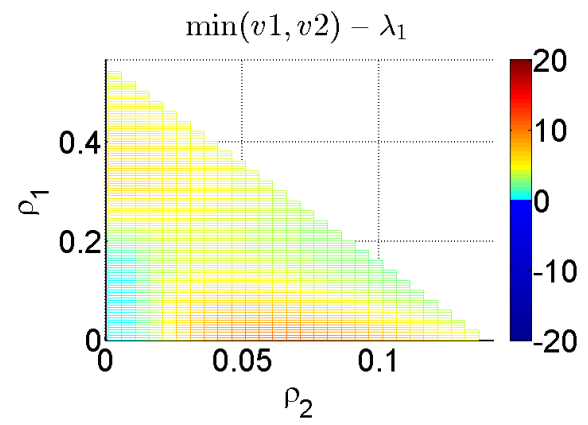

(a) $\min \left(V_{1}, V_{2}\right)-\lambda_{1}$

Figure 9: Evaluation of minimum characteristics speed over a point in $S=\left\{\rho_{1}, \rho_{2}\right\}$, Here $V_{1}=22 \mathrm{~m} / \mathrm{s}$ and $V_{2}=27 \mathrm{~m} / \mathrm{s}$

The results from the graphical analysis strongly suggest that the relation in equation (14) is valid for our model, which confirms that in the model no wave travels at a higher speed than the traffic and thus the wave propagation speed is finite.

\subsection{Model discretization}

To simulate the traffic flow we need the solution of the traffic equation in Eq. (3). Thus, we apply a conservative finite volume method for the approximation of the numerical solution. In the approximation, the spatial domain is divided into equal grid cells of size $\Delta x$ and at each time interval $\Delta t$ the density value in the domain is updated according to the conservation law. Rewriting in the integral form it becomes

$$
\frac{d}{d t} \int_{x_{i-1 / 2}}^{x_{i+1 / 2}} \rho(x, t) d x=q\left(\rho\left(x_{i-1 / 2}, t\right)\right)-q\left(\rho\left(x_{i+1 / 2}, t\right)\right)
$$

Integrating eq. (15) in time from $t^{n}$ to $t^{n+1}=t^{n}+\Delta t$, we have

$$
\begin{aligned}
\int_{x_{i-1 / 2}}^{x_{i+1 / 2}} \rho\left(x, t^{n+1}\right) d x= & \int_{x_{i-1 / 2}}^{x_{i+1 / 2}} \rho\left(x, t^{n}\right) d x \\
& +\int_{t^{n}}^{t^{n+1}} q\left(\rho\left(x_{i-1 / 2}, t\right)\right) d t-\int_{t^{n}}^{t^{n+1}} q\left(\rho\left(x_{i+1 / 2}, t\right)\right) d t .
\end{aligned}
$$


After some rearrangement of Eq. (16), we obtain an equation that relates cell average density $\rho_{j}^{n}$ update with average flux values at the cell interfaces.

$$
\rho_{i}^{n+1}=\rho_{i}^{n}-\frac{\Delta t}{\Delta x}\left[F_{i+1 / 2}^{n}-F_{i-1 / 2}^{n}\right],
$$

where $F_{i+1 / 2}^{n}$ is an average flux value at the cell interface $x=x_{i+1 / 2}$ :

$$
F_{i+1 / 2}^{n}=\mathcal{F}\left(\rho_{i}^{n}, \rho_{i+1}^{n}\right), \quad \text { where } \mathcal{F} \text { is the numerical flux function. }
$$

Accordingly, equation (17) rewrites

$$
\rho_{i}^{n+1}=\rho_{i}^{n}-\frac{\Delta t}{\Delta x}\left[\mathcal{F}\left(\rho_{i}^{n}, \rho_{i+1}^{n}\right)-\mathcal{F}\left(\rho_{i-1}^{n}, \rho_{i}^{n}\right)\right] .
$$

In the absence of a general Riemann solver, numerical methods for multi-class LWR model based on a generalization of the cell transmission model (CTM) supply and demand functions for each vehicle class have been introduced in (van Wageningen-Kessels, 2013; Fan and Work, 2015). However, these algorithms are computationally expensive to implement in our case, due to the lack of analytical expression for computing the numerical flux. Therefore, we have opted for the Lax-Friedrichs scheme (LeVeque, 1992), which is easier to implement and gives a good accuracy at sufficiently refined meshes. The numerical flux function is therefore given by

$$
\mathcal{F}\left(\rho_{i}, \rho_{i+1}\right)=\frac{1}{2}\left(q\left(\rho_{i}\right)+q\left(\rho_{i+1}\right)\right)+\frac{\alpha}{2}\left(\rho_{i}-\rho_{i+1}\right),
$$

where $\alpha$ is the numerical viscosity satisfying the condition $\alpha \geq V_{\max }=$ $\max \left\{v_{1}^{f}, v_{2}^{f}\right\}$. The space and time steps $\Delta x$ and $\Delta t$ are selected to meet Courant, Friedrichs and Lewy (CFL) condition, which is a necessary condition for a numerical method to achieve stability and convergence. Therefore, $\Delta t$ is chosen to satisfy $\Delta t \leq \Delta x / V_{\max }$, due to the bounds on the eigenvalues derived in Section 2.3.

\section{Model Verification}

The verification experiments are intended to evaluate our proposed model against the baseline model in (Nair et al., 2011), and the required qualitative behaviors. 


\subsection{Pore size distribution verification}

Here, we verify the pore size distribution against the results in (Nair et al., 2011), which are produced by determining the cumulative distribution of the pore size from the average of multiple simulation outcomes. We expect that the vehicle spacing distribution we propose yields qualitatively the same result as multiple simulation runs. To derive the pore size distribution, we have introduced simplification assumptions which are not used in (Nair et al., 2011). The impact of these assumptions on the model behavior can be grasped through the qualitative comparison between the results from our model and (Nair et al., 2011).

Therefore, we reproduce the result in (Nair et al., 2011) following the same approach used in the paper. In Nair's approach, for each configuration, the fraction of accessible pores is determined by running multiple simulation run, where vehicles are randomly placed in the domain (without overlapping) and then the probability of finding a pore greater than the critical pore size is determined from this configuration. However, at high density it may not be possible to find a solution within a reasonable amount of time. In these cases, the author proposed to adjust the pore space distribution to reflect 'unplaced vehicles'. But, nothing is mentioned in the paper how the pore space distribution can be adjusted. Thus, we applied our own method for adjusting the pore size distribution. For a given total number of vehicles, first the fraction of accessible pore $\left(F_{c}\right)$ is determined according to the 'placed vehicles'. If all the vehicles can not be placed within the time limit set, $F_{C}$ will be reduced by a ratio of total number of 'placed vehicles' to total number of vehicles.

For the sake of comparison, we use similar loading profile and simulation parameters. With normal profile, the interaction of the two classes under uninterrupted flow conditions is studied, while a traffic flow with disruption is studied in queue profile. The maximum speed is set to $V_{1}=80 \mathrm{~km} / \mathrm{hr}$ for PTWs and $V_{2}=100 \mathrm{Km} / \mathrm{hr}$ for cars. The simulation is done for $300 \mathrm{~s}$ on the space domain $x \in[0,3000 m]$, and with homogeneous initial density of $\rho_{1}(x, 0)=0, \rho_{2}(x, 0)=0$. We also set $\Delta x=100 \mathrm{~m}$ and $\Delta t=2.5 \mathrm{sec}$. For both experiments the upstream inflow is set to:

$$
F_{1}(0, t)= \begin{cases}0.5 v e h / s e c & \text { for } t \in[100 s, 200 s] \\ 0 & \text { otherwise }\end{cases}
$$


434

$$
F_{2}(0, t)= \begin{cases}0.5 v e h / s e c & \text { for } x \in[0 s, 200 s] \\ 0 & \text { otherwise }\end{cases}
$$

and we give absorbing boundary conditions downstream, so that the vehicles leave freely.

From Figure 10, it can be observed that PTWs traffic density wave moves faster than cars. Due to this, although PTWs starts behind, they move past cars traffic and leave the simulation domain faster. At $t=250 \mathrm{sec}$, all PTWs have overtaken cars. Both models behave similarly except small quantitative changes.

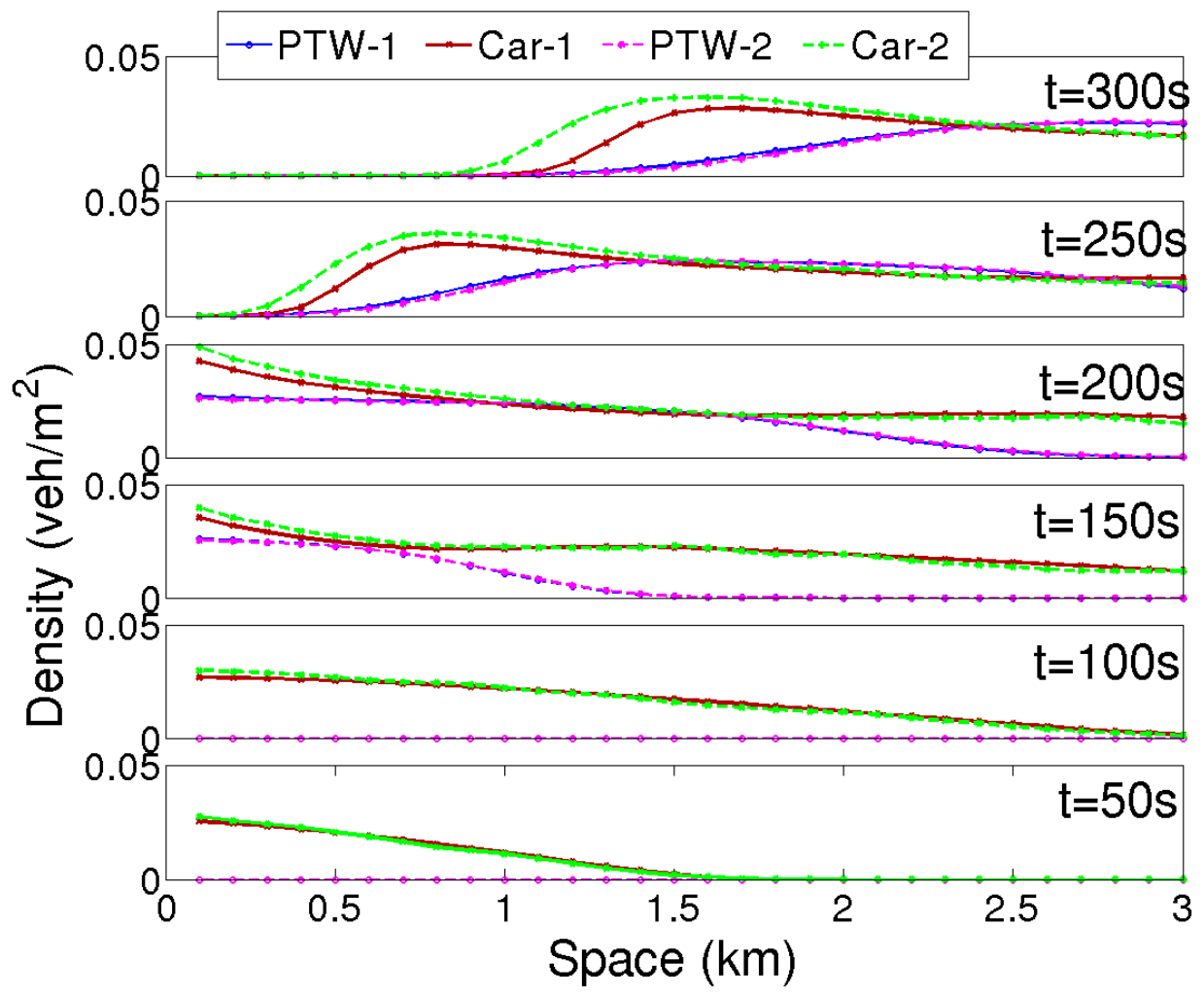

Figure 10: Normal profile, traffic density waves of cars and PTWs at different time steps. (PTW-1, Car-1) and (PTW-2, Car-2) represent result form our model and Nair's model, respectively. 


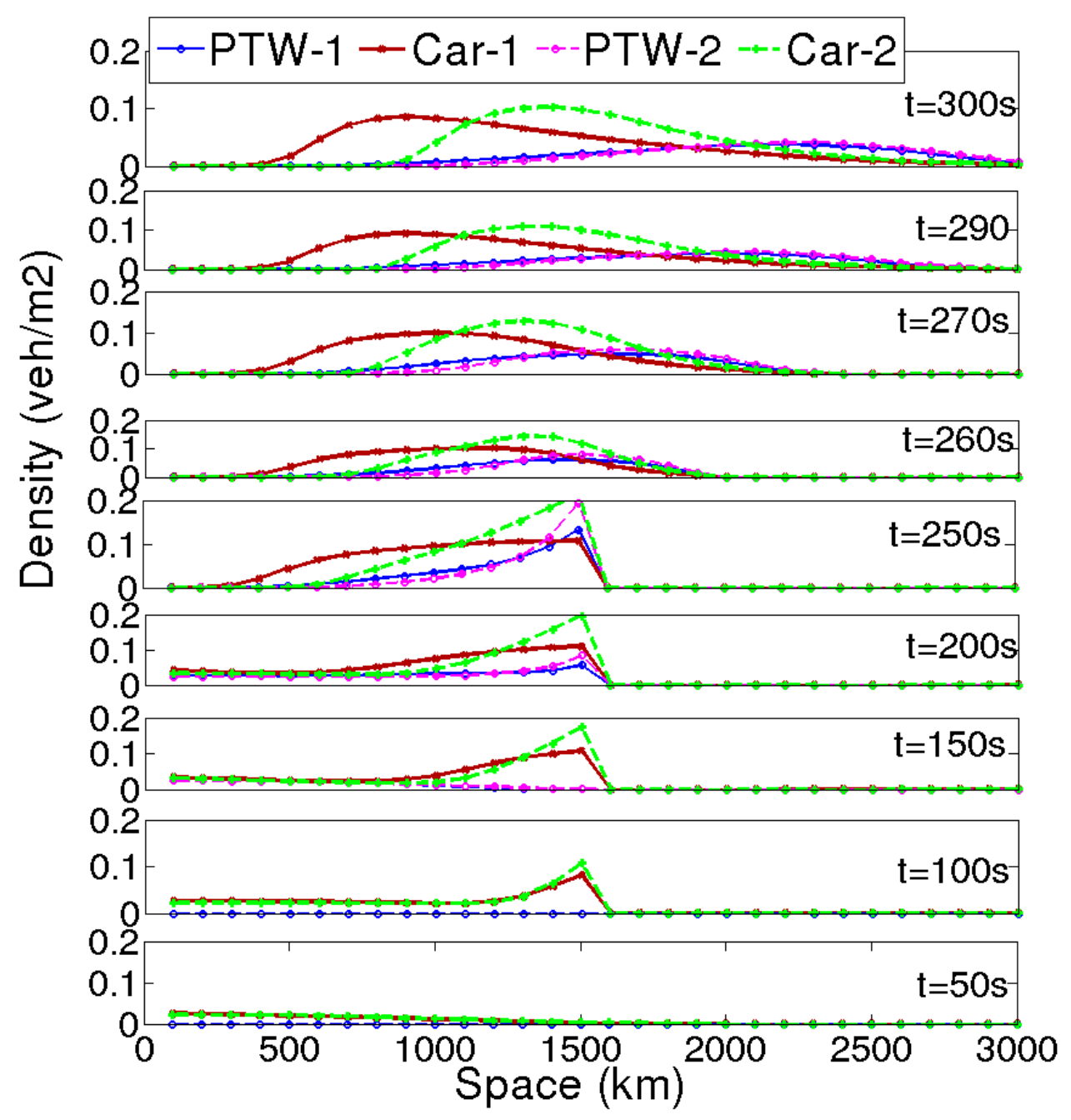

Figure 11: Queue profile, traffic density waves of cars and PTWs at different time steps. (PTW-1, Car-1) and (PTW-2, Car-2) represent result form our model and Nair's model, respectively.

The result in Figure 11 represents the interrupted scenario, where for $t \in[0 \mathrm{sec}, 250 \mathrm{sec}]$ the flow is blocked at the mid of roadway (at $1500 \mathrm{~m}$ ). Important properties observed from the results are: PTWs are able to move to the front of the queue passing stationary cars (from $t=200 \mathrm{sec}$ to $t=250 \mathrm{sec}$ ), thus, when the blockage is removed, PTWs clear first. In this scenario, a big quantitative divergence is observed between the two models, particularly 


$$
\text { (1) }
$$

when the queue is formed. In our model, we defined jam densities for each class and the speed function is scaled to reach zero at the jam densities (section 2.1, Figure 7). But, this modification is not applied to the speed function in Nair's model, see Figure 12. The difference between the speed values becomes more significant at the higher densities. The resulting quantitative change mainly happens because of the speed difference. Otherwise, both models are quantitatively similar.

The results in Figures 10 and 11, have almost the same qualitative properties as the results in (Nair et al., 2011), confirming the validity of the assumptions made to establish the distribution function of inter-vehicle spacing.

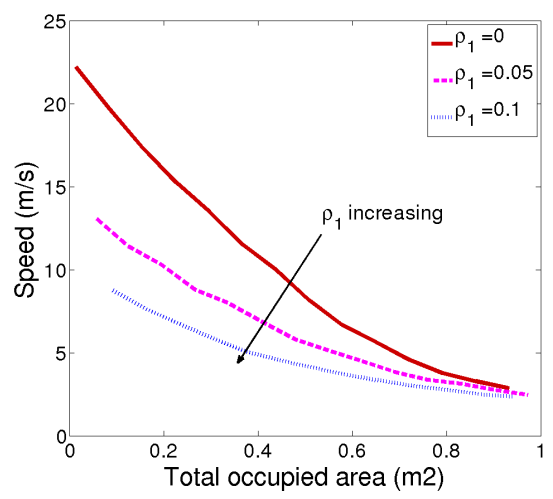

(a) Car speed at different density of (b) PTWs speed at different cars PTWs

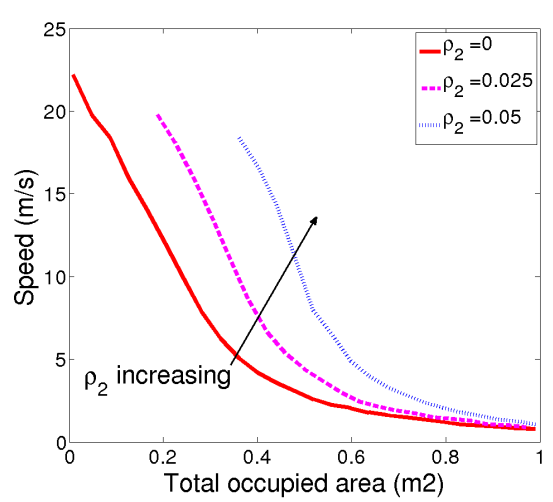

density values.

Figure 12: Speed Vs total occupied area $\left(\sum \rho_{1} A_{1}+\sum \rho_{2} A_{2}\right)$ Nair's model (Nair et al., 2011), where $\rho_{1} A_{1}$ and $\rho_{2} A_{2}$ are area projected on the road by PTW and car, respectively.

458

\subsection{Verifying model properties}

In this section, the capability of our model to reproduce the observed macroscopic phenomena of mixed flow of PTWs and cars is evaluated. The following two well-known features (Fan and Work, 2015) are used as a benchmark to evaluate our model.

- Overtaking- when the traffic volume is high, cars start slowing down. However, PTWs remain unaffected or less affected by the change in traffic situation, as they can ride between traffic lanes. As a consequence, PTWs travel at higher speed and overtake slow moving cars. 
- Creeping- when cars are stopped at traffic signals or because of traffic jams, PTWs can find a space to filter (creep) through stationary cars and move ahead.

In addition, a comparison with the models in (Benzoni-Gavage and Colombo, 2003) and (Fan and Work, 2015), hereafter referred as N-pop and creeping respectively, is presented along with the verification of our model, porous $G$.

For creeping and overtaking experiments, the parameters in Table 2 are chosen. Jam density refers to the maximum area occupancy, which equals to

\begin{tabular}{lcl} 
& PTW & Car \\
\hline \hline Vehicle length $(\mathrm{m})$ & 1.5 & 3 \\
Vehicle radius $(\mathrm{m})$ & 0.75 & 1.5 \\
Max. speed $(\mathrm{m} / \mathrm{s})$ & 1.8 & 1 \\
Jam density porous $G$ & 1 & 0.85 \\
Jam density creeping & 1.8 & 1 \\
Jam density $N$-pop & 1 & 1 \\
\hline
\end{tabular}

Table 2: Simulation Parameters

$\rho_{1} A_{1}+\rho_{2} A_{2}$ for porous $G$ model and $\rho_{1} l_{1}+\rho_{2} l_{2}$ for the other models, where vehicles come to complete stop state. The simulation is done on a road of length $50 \mathrm{~m}$ and $\Delta x=0.05 \mathrm{~m}$ and $\Delta t$ is selected according to CFL condition.

\subsubsection{Creeping experiment}

A signalized intersection is employed for testing creeping. In the simulation, PTWs start behind the cars traffic and cars traffic have concentrated close to the traffic signal, so that PTWs arrive after most of the cars reached a complete stop. The simulation is done for $200 \mathrm{sec}$ and starts with initial densities

$\rho_{1}(x, 0)=\left\{\begin{array}{ll}0.25 & \text { for } x \in[1 m, 21 m], \\ 0 & \text { otherwise },\end{array} \rho_{2}(x, 0)= \begin{cases}0.25 & \text { for } x \in[31 m, 50 m] \\ 0 & \text { otherwise }\end{cases}\right.$ The inflow and outflow at the boundaries are set to zero. At the time PTWs start catching up cars traffic (Figure 13(a)), most of the cars are at stationary state (see Figure 13(a) lower subplot space location $45-50 \mathrm{~m}$ ). However, as shown in Figure 13(b), PTWs maneuver through those stationary cars and 

a non-zero speed value even though cars are at a complete stop state.

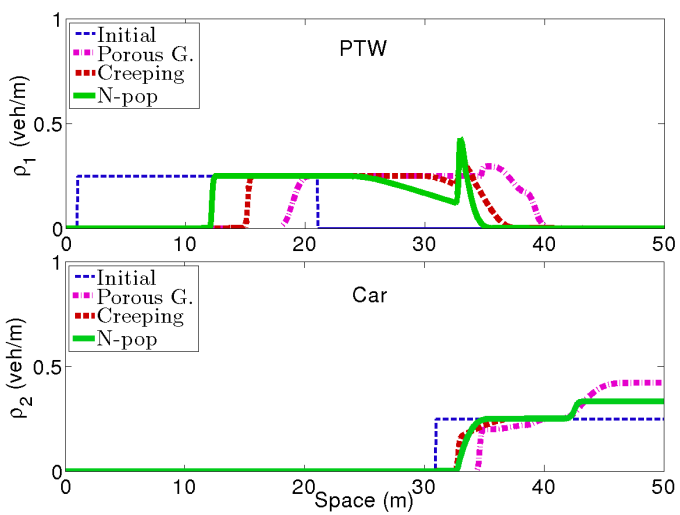

(a) Density profiles at time $t=10 \mathrm{~s}$.

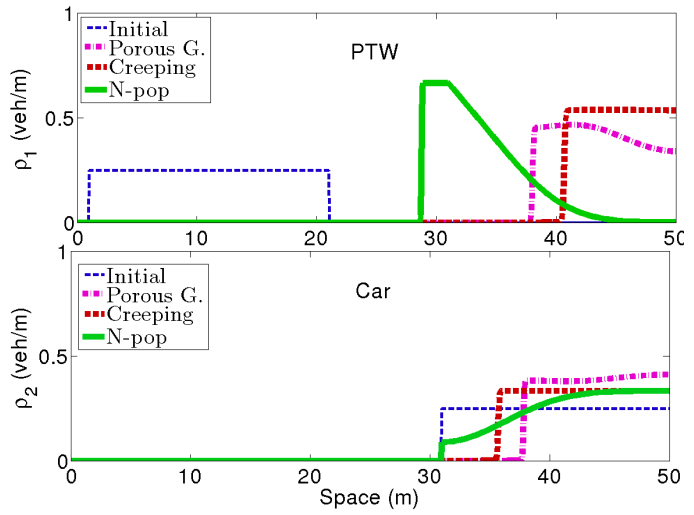

(b) Density profiles at time $\mathrm{t}=200 \mathrm{~s}$. reach the front of the queue for the case of creeping and Porous $G$ modces have the same jam density. Table 3 shows the average speeds of PTWs and cars in a particular location at time $t=50 \mathrm{~s}$. As can be observed from the speed values, unlike $N$-pop model, in the other two models PTWs have

Figure 13: Creeping experiment density-space diagram, upper subplot for PTWs and lower subplot for cars.

497

\begin{tabular}{cccc} 
& Creeping & Porous $G$ & N-pop \\
\hline \hline$V_{1}$ & 0.2179 & 0.6349 & 0 \\
$V_{2}$ & 0 & 0 & 0 \\
\hline
\end{tabular}

Table 3: Speed values extracted at time $t=50 \mathrm{sec}$ and position $x=39.15 \mathrm{~m}$

The results from the creeping experiment show similar behavior to the situation we may observe in real scenarios, i.e. PTWs seep through cars queue to reach the head the queue, both for Porous $G$ and Creeping models. However, for the $N$-pop model, PTWs remain behind car traffic queue. Thus, only the first two models are able to produce this predominantly observed phenomenon of mixed traffic flow of cars and PTWs.

\subsubsection{Overtaking experiment}

For the overtaking scenario, car traffic is placed ahead of PTWs. The simulation starts with the initial state where: 
507

$\rho_{1}(x, 0)=\left\{\begin{array}{ll}0.3 & \text { for } x \in[1 m, 20 m], \\ 0 & \text { otherwise, }\end{array} \rho_{2}(x, 0)= \begin{cases}0.3 & \text { for } x \in[15 m, 34 m] \\ 0 & \text { otherwise }\end{cases}\right.$

The inflow at the upstream boundary is set to zero and vehicles are allowed to leave freely at the downstream boundary. For this experiment, we consider two cases one when free flow speed of PTWs is higher than cars and the other when cars take the higher free flow speed. The occurrence of overtaking is evaluated by inspecting the evolution of traffic densities of the two classes. Overtaking is said to happen when the density waves of the two classes come to the same level in space and one of the two go past the other, i.e the tail end of one class is before the other.

As Figure 14 depicts, when free flow speed of PTWs is greater than cars, PTWs overtake cars in all the three models. In Porous $G$ model overtaking is observed around at time $t=18 \sec$ (Figure 14(b)), and for Creeping and $N$-pop models overtaking happens at $t=38 \mathrm{sec}$ (Figure $14(\mathrm{c})$ ) and $t=80 \mathrm{sec}$ (Figure 14(d)), respectively.

The simulation results in Figure 15 correspond to the case where free flow speed of cars $\left(V_{2}=1.8\right)$ is greater than free flow speed of PTWs $\left(V_{1}=1.5\right)$. As shown, in the two models, Porous $G$ and Creeping, overtaking is observed. In Porous $G$ model overtaking happens around time $t=26 \sec$ (Figure 15(b)) and at time $t=40 \sec$ (Figure 15(c)) for Creeping. Nonetheless, $N$-pop model fails to reproduce overtaking. At time $\mathrm{t}=52 \mathrm{sec}$ for $\mathrm{N}$-pop the tail end of PTWs traffic is around location $x=26 m$ whereas for cars traffic it is around $x=41 m$ (Figure15(d)), which is far behind.

According to what is illustrated in Figures 14 and 15, all the three models are able to show the overtaking phenomenon when PTWs free flow speed is higher than cars. Further, for Porous $G$ and Creeping models overtaking happens in the case where free flow speed of cars is higher than PTWs' as well. In $N$-pop model, unlike to the other two models, overtaking never happens unless car free flow speed is higher. This can be explained using a particular instance in Figure 16. As shown in the figure, in Creeping and Porous $G$ models there exist a region where the speed of PTWs is greater than cars despite the free flow speed choice.

In conclusion, the model verification results validate that our model (Porous $G$ ) can reproduce the required creeping and overtaking phenomena.The Creeping model also satisfies all these properties. Yet, this model has a limitation, as occupied space is a mere factor that determines the speed and the varia- 


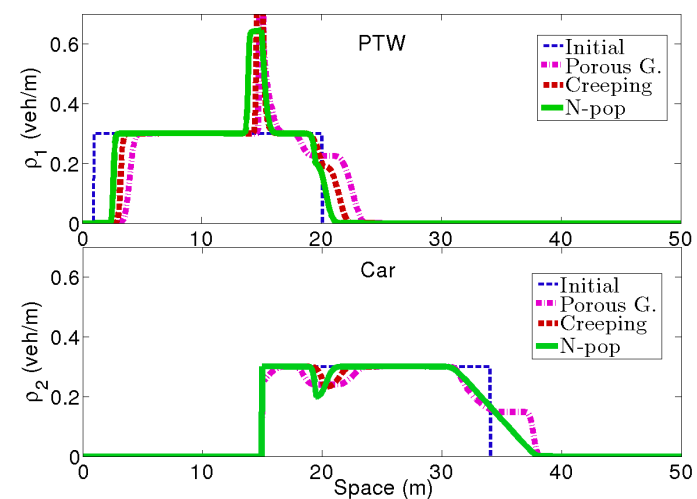

(a) Density profiles at time $t=2 \mathrm{sec}$.

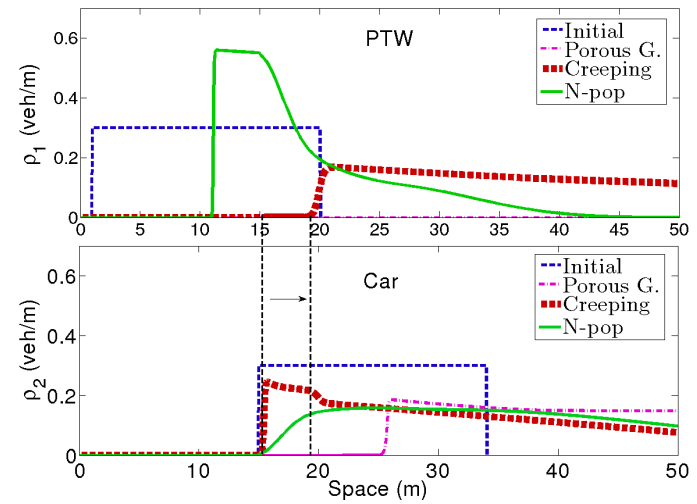

(c) At ime $t=38 \mathrm{sec}$, overtaking in Creeping.

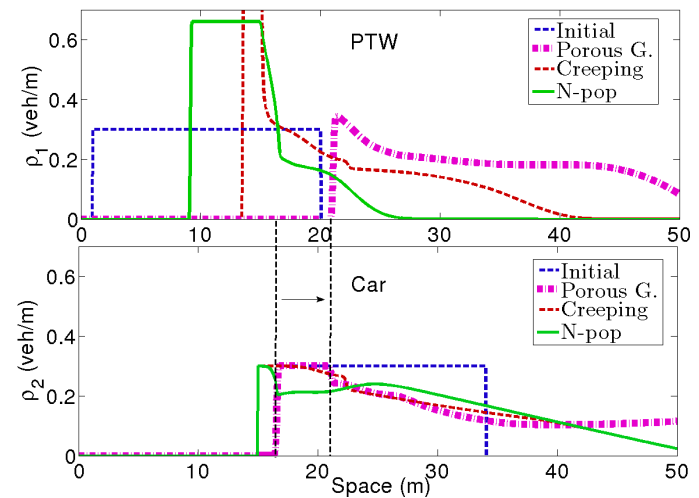

(b) At time $\mathrm{t}=18 \mathrm{sec}$, overtaking in Porous G

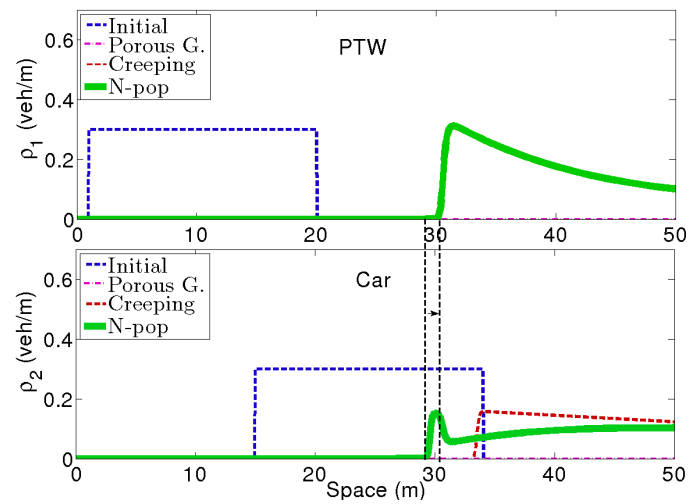

(d) At time $\mathrm{t}=80 \mathrm{sec}$, overtaking in N-pop.

Figure 14: Overtaking experiment density-space diagrams, upper subplot for PTWs and lower subplot for cars, free flow speed of $V_{1}=1.8 \mathrm{~m} / \mathrm{s}$ greater than $V_{2}=1 \mathrm{~m} / \mathrm{s}$. The dashed lines stretching from upper subplot to the lower connect the tail of the density profiles for cars and PTWs' traffic and the spacing between the two lines indicates the distance gap after PTWs overtake. 


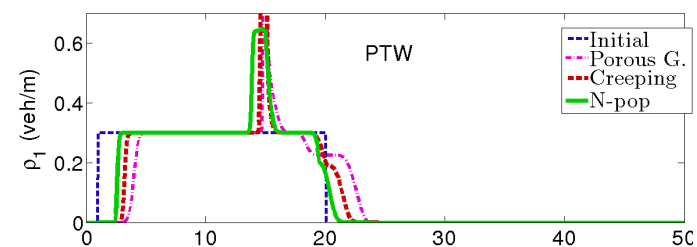

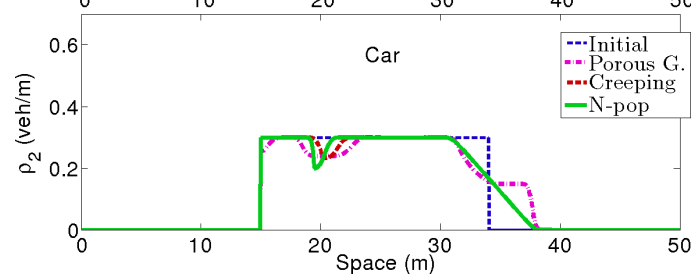

(a) Density profiles at time $t=2$ sec.

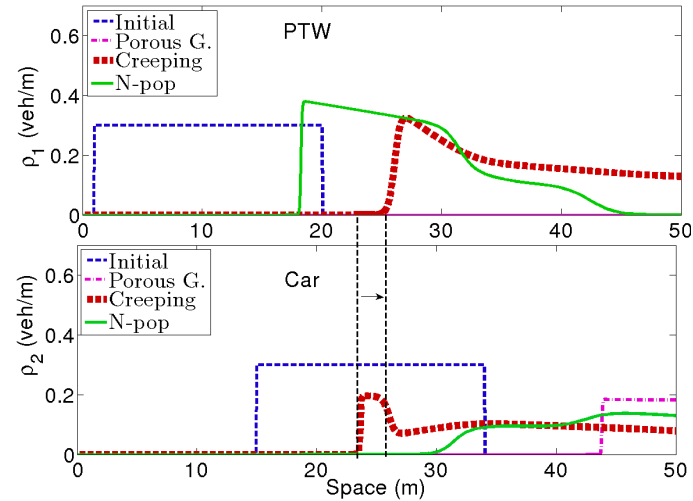

(c) At time $\mathrm{t}=40 \mathrm{sec}$, overtaking in Creeping.

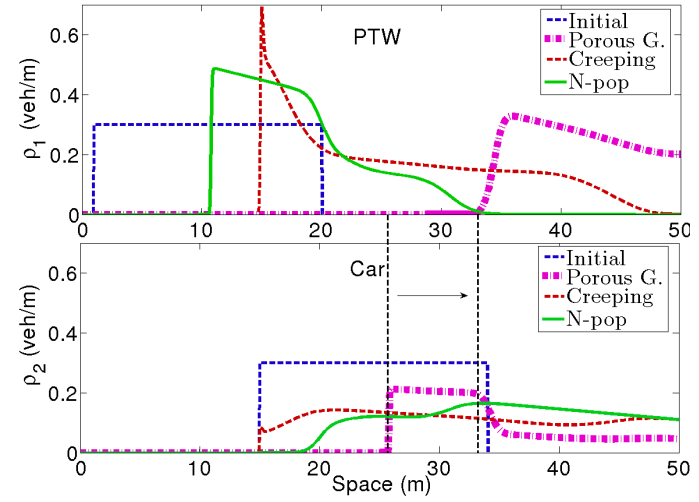

(b) At time $\mathrm{t}=26 \mathrm{sec}$, overtaking in Porous G.

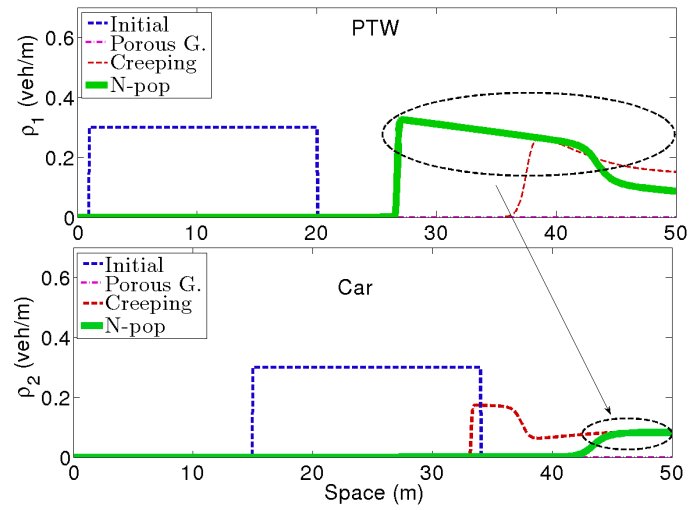

(d) At time t=52sec, $N$-pop.

Figure 15: Overtaking experiment density-space diagrams, upper subplot for PTWs and lower subplot for cars, free flow speed of $V_{2}=1.8 \mathrm{~m} / \mathrm{s}$ greater than $V_{1}=1.5 \mathrm{~m} / \mathrm{s}$. 


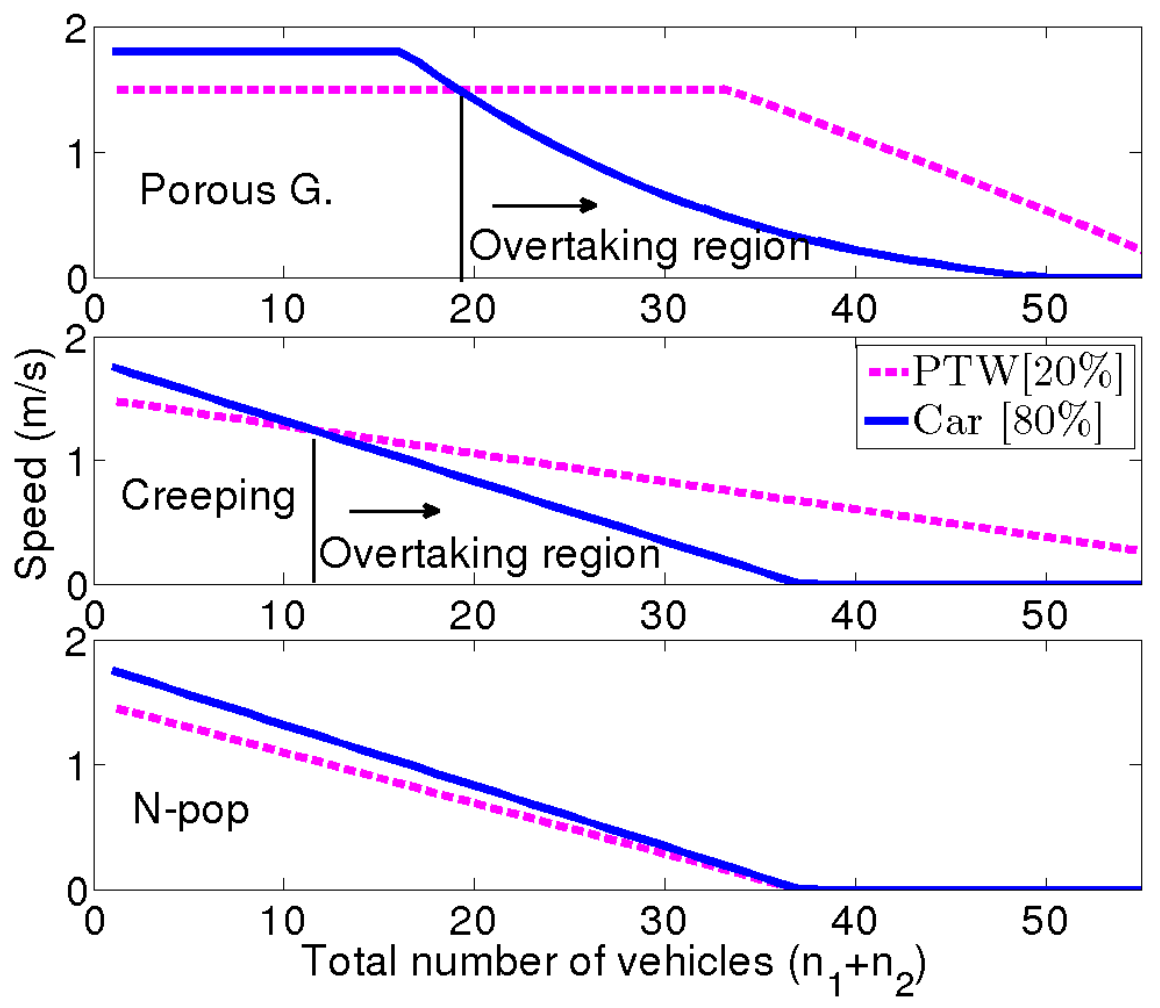

Figure 16: Speed vs. total number of vehicles plot, when free flow speed of PTWs less than cars and cars account to $80 \%$ of the total traffic, upper subplot Porous $G$, middle subplot Creeping, lower subplot $N$-pop. 
tion in the composition of vehicles has no influence as long as the occupied space is the same (see section 2.1, Figure 7). The $N$-pop model, however, lacks the creeping behavior and overtaking is conditioned by the free flow speed of PTWs.

\section{Traffic impact analysis}

The traffic impact analysis aims to assess the potential improvements in traffic mobility obtained from growing use of PTWs. Identifying the opportunities leads to the introduction of new innovative smart city applications. Furthermore, it gives the necessary information on how transport policies, mobility plan, traffic management, etc. should be shaped to benefit from the opportunities. Thus, the section here explores the impact of PTWs on traffic flow, road capacity and queue discharge time. First, we analyze the role of PTWs, at different penetration rates, on minimizing congestion, by substituting some of the cars with PTWs. Next, we investigate how shifting travel mode to PTWs could help in the reduction of travel times. Finally, we study the effect of PTWs filtering behavior on queue discharging time.

\subsection{Road capacity}

Road capacity, which is also called critical density, is defined as the maximum volume of traffic that corresponds to the maximum flow rate. Above the road capacity, traffic flow enters congestion state and the flow of vehicles decreases with the increase in traffic volume. In mixed traffic flow, the road capacity varies depending on the total density and the traffic composition. Here, the role of PTWs in reducing congestion is evaluated. For the comparison, the flow-density plot for different ratios of PTWs is presented in Figure 17. The following simulation parameters are used to produce the results. The maximum speed of cars is $V_{2}=100 \mathrm{~km} / \mathrm{hr}$, maximum speed of PTWs is $V_{1}=80 \mathrm{~km} / \mathrm{hr}$ and we consider a single lane one-way road with a carriage width of $3.5 \mathrm{~m}$.

PTWs stay in free flow state for longer ranges of density than cars, because of their ability to ride in between other vehicles. The flow-density diagram, which is depicted in Figure 17(b), shows the variation of maximum flow rate and critical density of the two classes. Figure 17(a) shows the total flow rate against the total volume of vehicles. The total flow rate describes the number of vehicles that leave a given point per unit time, which in our case is equal to the sum of the flow rates of PTWs and cars. As Figure 17(a) 
illustrates, increasing the proportion of PTWs on the total traffic from $0 \%$ to $10 \%$ results in a $9.3 \%$ improvement of the road capacity and $2.74 \%$ of the maximum flow rate. The results in Figure 17 and Table 4 point up that shift

\begin{tabular}{lcl} 
\% of PTWs & $\begin{array}{c}\text { Critical density } \\
\text { (veh/km) }\end{array}$ & $\begin{array}{l}\text { Maximum flow } \\
\text { (veh/hr) }\end{array}$ \\
\hline \hline 0 & 43.1 & 4248 \\
10 & 47.1 & 4320 \\
25 & 58.1 & 4608 \\
35 & 72.1 & 4896 \\
50 & 116.1 & 6084 \\
\hline
\end{tabular}

Table 4: The Change in Critical Density (veh/ $\mathrm{km}$ per unit lane width) and Maximum Flow Rate (veh/hr/lane) at Different Ratios of PTWs

to PTWs indeed helps to improve road capacity. Besides, the variation on the reaction of the two traffic classes for a given traffic situation entails a new method for mobility management and monitoring.

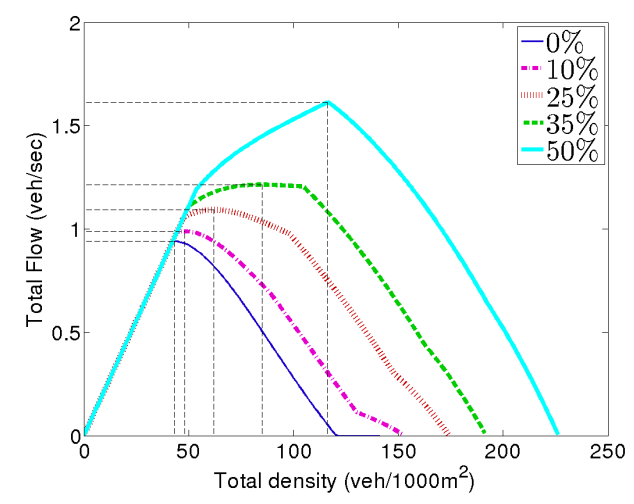

(a) Total flow rate vs. total density,the connecting dashed lines show the maximum flow rate and the corresponding road capacity.
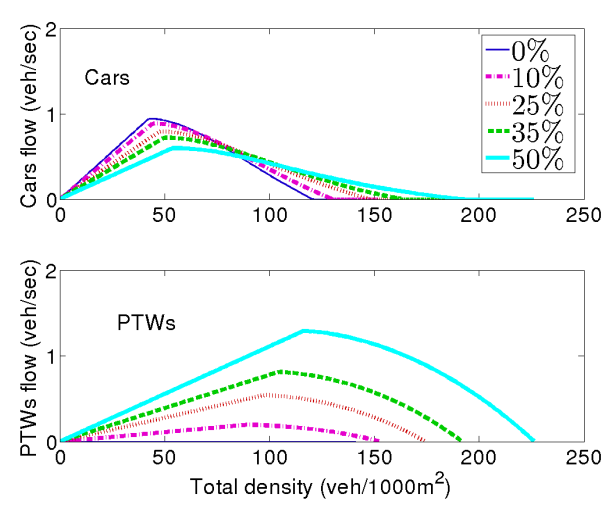

(b) Flow-total density diagram, upper subplot for cars and lower subplot for cars.

Figure 17: Flow-density diagram,for different penetration rates of PTWs.

\subsection{Travel time}

Here, we analyze how replacing some of the cars with PTWs improves travel time based on the instantaneous travel time analysis. The instanta- 
neous travel time (iTT) is computed on the assumption that vehicles travel through the considered road section at a speed profile identical to that of the present local speed and it is formulated as:

$$
t_{\text {inst }}=\sum_{i=1}^{n} \frac{\Delta x}{v\left(x_{i}, t\right)},
$$

where $n$ is the number of cells and $\Delta x$ is the mesh size. The experiment is done under the following simulation setups: road length $500 m, \Delta x=$ $10 \mathrm{~m}$, free flow speeds $V_{1}=V_{2}=80 \mathrm{~km} / \mathrm{hr}$ and the simulation is run for 80sec. A homogeneous initial total density of $\rho_{1}(x, 0)+\rho_{2}(x, 0)=0.1$ for $x \in[0,500 m]$ is set. The result in Figure 18 is produced by computing the instantaneous travel time every $0.02 \mathrm{sec}$. According to the result, a $12.4 \%$ reduction on average travel time is obtained even at the lowest penetration of PTWs (10\%). The table in Figure 18 below presents the iTT values averaged over the whole simulation period for different traffic compositions and the improvement on the average travel time. According to these results, in addition to the reduction of the average travel times, with more shift of cars to PTWs, cars travel at high speed for more time. Certainly, the results show that PTWs help in maintaining reliable and reduced travel times.

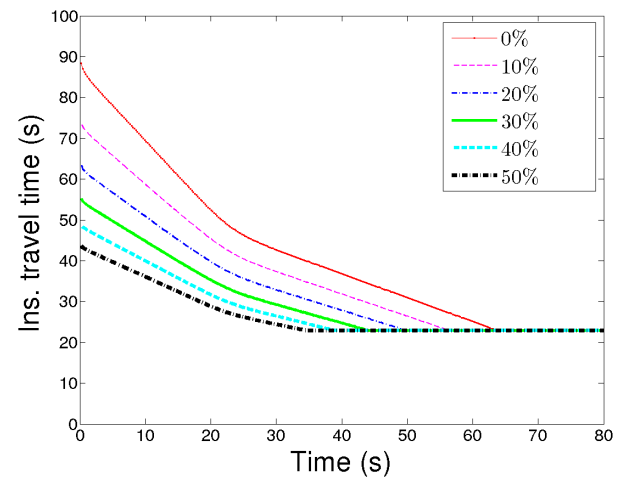

\begin{tabular}{ccc}
$\begin{array}{c}\text { \% of } \\
\text { PTWs }\end{array}$ & $\begin{array}{c}\text { cars average } \\
\text { travel time }\end{array}$ & $\begin{array}{c}\text { Improv. } \\
(\%)\end{array}$ \\
\hline \hline 0 & 41.6 & \\
10 & 36.45 & 12.4 \\
20 & 32.74 & 21.3 \\
30 & 30 & 27.9 \\
40 & 28 & 32.7 \\
50 & 26.68 & 35.9 \\
\hline
\end{tabular}

Figure 18: Change in travel time of cars for different penetration rate of PTWs.

\subsection{Queue clearance time}

At signalized intersections, PTWs creep through the queue of other traffic to reach the front line. As more PTWs accumulate at the front of the queue, it is likely that they discharge from the queue much quicker than cars. Since 


\section{critical pore size for both classes.}

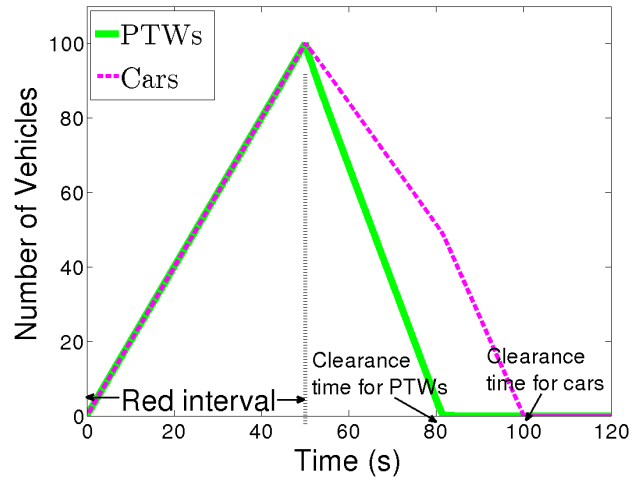

(a) Clearance time when Filtering of PTWs is allowed.

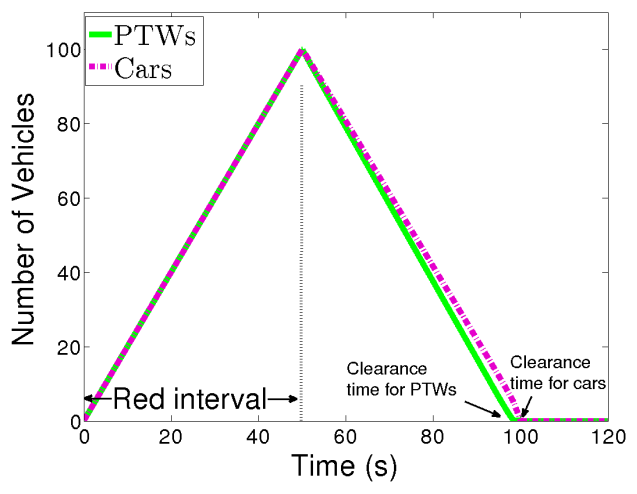

(b) Clearance time, no filtering.

Figure 19: Evolution of number of vehicles in the queue over time. 


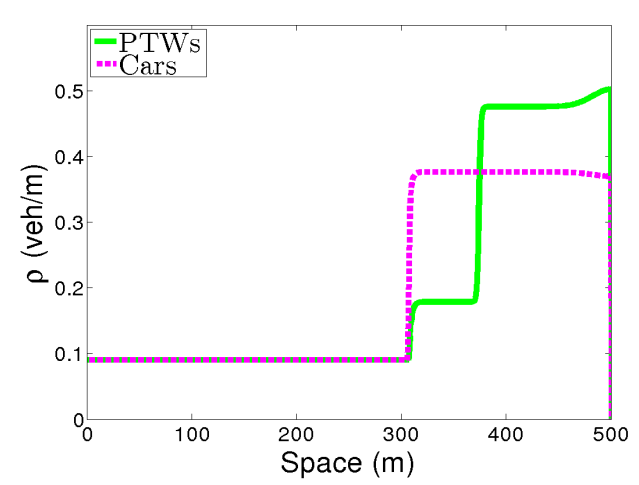

(a) Density profile of PTWs and cars, when PTWs are allowed to filter through traffic queue.

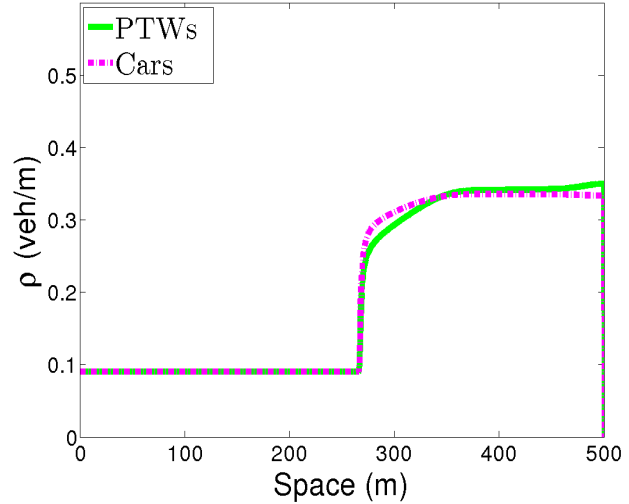

(b) Density profile of PTWs and cars, cars and PTWs behave in a similar manner.

Figure 20: Spatial distribution of the density of vehicles in the queue.

The simulation are run on the space domain $x \in[0,5001 \mathrm{~m}]$ and the inflow in the upstream direction, for both classes, is set to have the following values:

$$
F_{i}(0, t)= \begin{cases}2 v e h / s & \text { for } t \in[0,50 \mathrm{sec}] \\ 0 & \text { otherwise. }\end{cases}
$$

The traffic light (TL) is placed at $x=500 \mathrm{~m}$. The simulation starts with a red phase and stays in this state for the first 50 seconds.

To observe the queue clearance time and queue discharging behavior for both vehicle classes, the evolution of the number of vehicles in the queue is shown in Figure 19 and the spatial distribution of vehicles in the queue, immediately before the beginning of the green light period, is presented with the density profile plot shown in Figure 20.

According to the results from the first experiment, where filtering of PTWs allowed, most of the PTWs occupy the front of the queue during the queue formation (see Figure 20(a)), and they clear from the queue $28 \mathrm{sec}$ before cars traffic. On the other hand, no difference is observed in the clearance time of the two classes when PTWs are forced to behave in a similar manner to cars.

A comparison of the plots in Figure 20(a) with Figure 20(b) show that, with the filtering of PTWs, higher percentage of PTWs reach the front line 
of the queue. However, PTWs attain high speed rapidly and dissipate from the queue faster. As a result, there is no a significant delay incurred on cars traffic because of the filtering behavior of PTWs. The message here is that PTWs creeping behavior has no influence on clearance time of cars, but rather improves the average delay experienced by road users at the intersections. Having a facility which helps PTWs to leave first at the intersections would allow better use of this opportunity offered by PTWs.

In general, the results indicate the positive impact of PTWs creeping behaviors on queue clearance time and the necessity to consider such behaviors on the design of traffic light operation, particularly when the ratio of PTWs is higher.

\section{Calibration of the model}

The model is validated against the desired qualitative behaviors. Yet, to accurately reproduce the real traffic situation adjusting the model parameters is imperative. The model is founded on the assumption that the traffic flow behavior can be characterized using the inter-vehicle spacing distribution. Thusly, the accuracy of the model highly depends on how precisely the inter-vehicle spacing distribution is estimated. The inter-vehicle spacing distribution, therefore, has to be calibrated from empirical data. The calibration process involves, for different traffic compositions and densities, collecting position information of vehicles, measuring spacing between vehicles, estimating statistical parameters of inter-spacing (mean, variance) and curve fitting experiments. Thereafter, the functional relationship of speed and inter-vehicle spacing distribution should be calibrated based on real observation. This could be done by employing a trial and error calibration method where the value of the speed function parameters, such as critical pore size (gap) and jam density, are adjusted until a good fitting curve to the observation is obtained. The jam and critical density values are dependent on the actual traffic state, that is, the traffic compositions and density. Therefore, it is also necessary to establish an accurate relationship between the jam and critical density parameters, and the traffic state.

For the calibration, real trajectory data for each vehicle class and different ranges of density is required. In addition, for non-lane based traffic the influence of the road geometry is significant, thus information about the roadway such as lane width, number of lanes, etc is necessary. Although there are widely available methods to collect vehicles' trajectory data, only a few of 
them are applicable for the required validation experiment. The challenge is mainly on getting the required traffic parameters and accurate geo-location of vehicles, specifically PTWs. For example, data collected from sensors like inductive loops are not sufficient as extrapolation of vehicles spatial location is very difficult, if not impossible. Floating Car Data (FCD) could be an efficient method for collecting vehicles' trajectory data, where smartphones or GPS devices in vehicles continuously send location, speed, etc. information. However, the inefficiency of smartphone GPS to produce the true location of PTWs (Koyama and Tanaka, 2011) and the low penetration rate of vehicles equipped with an accurate GPS receiver make FCD method less applicable. Another potential alternative is to use video cameras and to extract the required traffic data (vehicle number, vehicle type, location, etc.) utilizing image processing techniques (Mallikarjuna et al., 2009). Given the complexity of data collection, calibrated commercial simulators like VISSIM can serve as a means of model calibration. Yet, as the simulator might be calibrated to a particular scenario, the model validation would be valid only to that specific scenario.

\section{Summary and conclusion}

Motorcycles, scooters and other moped, thereafter referred to as Powered Two-Wheelers (PTWs), have peculiar maneuvering behaviors, such as filtering through slow moving or stationary traffic, or lacking lane discipline, which create mixed traffic flow characteristics resembling more disordered flows rather than lane-based follow-the-leader flows. Mixed flow models considering ordered flows accordingly fail to truly represent the impact of PTW on heterogeneous traffic flow characteristics. This paper specifically investigated disordered PTWs moving similarly to a fluid in a porous medium. An enhanced mixed flow traffic model is provided, based on an innovative modeling of the distribution of the pore sizes. This model is then used to evaluate the impact of a gradual penetration of PTWs on mixed flow traffic characteristics.

The close form distribution of pore size in porous media has been validated by comparing it against typical PTW flow characteristics and also benchmarked against related studies. This model allowed us to propose a mathematical formulation of the fundamental relation between speed and density for both cars and PTW individually. The latter aspect could be very 
beneficial in related traffic flow studies, which assumed identical fundamental relations for PTWs and cars.

The evaluation of the impact of PTWs on mixed traffic showed that a gradual replacement of cars with PTWs manages to increase the flow capacity by $9.3 \%$ already with $10 \%$ PTW penetration. The results not only confirmed the benefit of PTWs in reducing travel times, but also illustrated the mutual benefit of a gradual penetration of PTWs on travel times for both PTWs and passenger cars $(12.4 \%$ benefit on cars at $10 \%$ penetration of PTWs). Finally, we also showed that PTWs creeping through slow passenger car traffic at traffic light actually impacts queue clearance time and as such should be considered by traffic light where the cycles length is set according to queue clearance time.

The presented model assumes that both classes of vehicles disregard the lane discipline and their spatial distribution over the road segment follows Poisson point process. As a future work, we aim to consolidate the model by applying a more realistic approach for the spatial distribution and lane discipline of cars. The model is validated against the desired qualitative behaviors. Yet, to accurately reproduce the real traffic situation adjusting the model parameters is imperative. The model parameters such as the maximum speeds, jam and critical densities, stochastic characteristics of the probability density function of the spacing distribution, and the fundamental diagram should be tuned using real traffic data. For the calibration, the traffic data collected either from field or calibrated simulation platforms can be used. Because of the scarcity of real traffic data containing the trace of PTWs, we will perform the model calibration using VISSIM, which is a calibrated simulation platform.

\section{References}

Adnan, M., 2014. Passenger car equivalent factors in heterogenous traffic environment-are we using the right numbers? Procedia engineering 77, 106-113.

Ambarwati, L., Pel, A. J., Verhaeghe, R., van Arem, B., 2014. Empirical analysis of heterogeneous traffic flow and calibration of porous flow model. Transportation research part C: emerging technologies 48, 418-436.

Benzoni-Gavage, S., Colombo, R. M., 2003. An n-populations model for traffic flow. European Journal of Applied Mathematics 14 (05), 587-612. 
Chanut, S., Buisson, C., 2003. Macroscopic model and its numerical solution for two-flow mixed traffic with different speeds and lengths. Transportation Research Record: Journal of the Transportation Research Board (1852), 209-219.

Chen, G., Meng, F., Fu, G., Deng, M., Li, L., 2013. A cell automation traffic flow model for mixed traffic. Procedia-Social and Behavioral Sciences 96, 1412-1419.

Daganzo, F. C., 2002. A behavioral theory of multi-lane traffic flow. part I: Long homogeneous freeway sections. Transportation Research Part B: Methodological 36, 131-158.

Fan, S., Work, D. B., 2015. A heterogeneous multiclass traffic flow model with creeping. SIAM Journal on Applied Mathematics 75 (2), 813-835.

Jiang, C., Zhang, H., Han, Z., Ren, Y., Leung, V. C., Hanzo, L., 2016. Information-sharing outage-probability analysis of vehicular networks. IEEE Transactions on Vehicular Technology 65 (12), 9479-9492.

Koyama, Y., Tanaka, T., 2011. High-precision motorcycle trajectory measurements using gps. SICE Journal of Control, Measurement, and System Integration 4 (3), 199-205.

Lenorzer, A., Casas, J., Dinesh, R., Zubair, M., Sharma, N., Dixit, V., Torday, A., Brackstone, M., 2015. Modelling and simulation of mixed traffic. In: Australasian Transport Research Forum (ATRF), 37th, 2015, Sydney, New South Wales, Australia.

LeVeque, R. J., 1992. Numerical methods for conservation laws. Vol. 132. Springer.

Lighthill, M., Whitham, G., 1955. On kinematic waves. i. flood movement in long rivers. In: Proceedings of the Royal Society of London A: Mathematical, Physical and Engineering Sciences. Vol. 229. The Royal Society, pp. 281-316.

Logghe, S., Immers, L. H., 2008. Multi-class kinematic wave theory of traffic flow. Transportation Research Part B: Methodological 42 (6), 523-541. 
Mallikarjuna, C., Phanindra, A., Rao, K. R., 2009. Traffic data collection under mixed traffic conditions using video image processing. Journal of transportation engineering 135 (4), 174-182.

Mallikarjuna, C., Rao, K. R., 2006. Area occupancy characteristics of heterogeneous traffic. Transportmetrica 2 (3), 223-236.

Mathew, T. V., Munigety, C. R., Bajpai, A., 2013. Strip-based approach for the simulation of mixed traffic conditions. Journal of Computing in Civil Engineering 29 (5), 04014069.

Miles, R. E., 1970. On the homogeneous planar poisson point process. Mathematical Biosciences 6, 85-127.

Minh, C. C., Sano, K., Matsumoto, S., 2012. Maneuvers of motorcycles in queues at signalized intersections. Journal of advanced transportation 46 (1), 39-53.

Nair, R., Mahmassani, H. S., Miller-Hooks, E., 2011. A porous flow approach to modeling heterogeneous traffic in disordered systems. Transportation Research Part B: Methodological 45 (9), 1331-1345.

Nair, R., Mahmassani, H. S., Miller-Hooks, E., 2012. A porous flow model for disordered heterogeneous traffic streams. In: Transportation Research Board 91st Annual Meeting. No. 12-3260.

Newell, G. F., 1993. A simplified theory of kinematic waves in highway traffic, part i general theory, part ii: Queueing at freeway bottlenecks, part iii multi-destination flows. Transportation Research Part B: Methodological 27 (4), 281-313.

Pandey, G., Rao, K. R., Mohan, D., ???? Modelling vehicular interactions for heterogeneous traffic flow using cellular automata with position preference. Journal of Modern Transportation, 1-15.

Praveen, P. S., Arasan, V. T., 2013. Influence of traffic mix on pcu value of vehicles under heterogeneous traffic conditions. International Journal of Traffic and Transport Engineering 3 (3), 302-330.

Richards, P. I., 1956. Shock waves on the highway. Operations research 4 (1), $42-51$. 
SHIOMI, Y., HANAMORI, T., Eng, M., Nobuhiro, U., SHIMAMOTO, H., 2012. Modeling traffic flow dominated by motorcycles based on discrete choice approach. In: Proceedings of 1st LATSIS Conference.

Van Lint, J., Hoogendoorn, S., Schreuder, M., 2008. Fastlane: New multiclass first-order traffic flow model. Transportation Research Record: Journal of the Transportation Research Board (2088), 177-187.

van Wageningen-Kessels, F., 2013. Multi-class continuum traffic flow models: analysis and simulation methods. Ph.D. thesis, TRAIL.

Wong, G., Wong, S., 2002. A multi-class traffic flow model-an extension of lwr model with heterogeneous drivers. Transportation Research Part A: Policy and Practice 36 (9), 827-841.

Zhang, H., Jin, W., 2002. Kinematic wave traffic flow model for mixed traffic. Transportation Research Record: Journal of the Transportation Research Board (1802), 197-204.

Zhang, P., Liu, R.-X., Wong, S., Dai, S.-Q., 2006. Hyperbolicity and kinematic waves of a class of multi-population partial differential equations. European Journal of Applied Mathematics 17 (2), 171-200.

\section{List of Figures}

1 Heterogeneous traffic flow for PTWs and cars. . . . . . . 7

2 Vehicles spacing distribution, where $\rho_{1}$ and $\rho_{2}$ represent, respectively, PTWs and cars density . . . . . . . . . . . 8

3 Delaunay triangle edges length for circles. . . . . . . . . 9

4 Comparison of estimated probability distribution function and fitting theoretical distributions for different vehicles composition 10

5 Example $\rho_{1}=\rho_{2}=0.03 \mathrm{veh} / \mathrm{m}^{2}$ : PDF of the inter-vehicle distance on a road with dimension $L=100$ and $W$ ranging from $5 m-100 m \ldots \ldots \ldots \ldots \ldots$

6 Speed vs total occupied area for constant critical pore size $\left(r_{c}^{2}=3 m\right)$ and a variable critical pore size $\left(r_{c}^{1}\right)$ with the following parameters $r_{c}^{\min }=3 m$ and $r=2 m . \ldots \ldots 13$ 
Speed Vs total occupied area $\left(\sum \rho_{1} A_{1}+\sum \rho_{2} A_{2}\right)$, where $\rho_{1} A_{1}$ and $\rho_{2} A_{2}$ are area projected on the road by PTW and car, respectively. . . . . . . . . . . . . . . . . . 14

8 Evaluation of the maximum characteristics speed over a point in $S=\left\{\rho_{1}, \rho_{2}\right\}$,Here $\mathrm{V}_{1}=22 \mathrm{~m} /$ sand $\mathrm{V}_{2}=27 \mathrm{~m} / \mathrm{s} \ldots \ldots 17$

$9 \quad$ Evaluation of minimum characteristics speed over a point in $S=\left\{\rho_{1}, \rho_{2}\right\}$, Here $\mathrm{V}_{1}=22 \mathrm{~m} /$ sand $\mathrm{V}_{2}=27 \mathrm{~m} / \mathrm{s} \ldots$. . . . . 18

10 Normal profile, traffic density waves of cars and PTWs at different time steps. (PTW-1, Car-1) and (PTW-2, Car-2) represent result form our model and Nair's model, respectively. . 21

11 Queue profile, traffic density waves of cars and PTWs at different time steps. (PTW-1, Car-1) and (PTW-2, Car-2) represent result form our model and Nair's model, respectively. . 22

12 Speed Vs total occupied area $\left(\sum \rho_{1} A_{1}+\sum \rho_{2} A_{2}\right)$ Nair's model (Nair et al., 2011), where $\rho_{1} A_{1}$ and $\rho_{2} A_{2}$ areareaprojectedontheroadbyPTWandcar, respect

13 Creeping experiment density-space diagram, upper subplot for PTWs and lower subplot for cars. . . . . . . . . . . . . 25

14 Overtaking experiment density-space diagrams, upper subplot for PTWs and lower subplot for cars, free flow speed of $V_{1}=$ $1.8 \mathrm{~m} / \mathrm{s}$ greater than $V_{2}=1 \mathrm{~m} / \mathrm{s}$. The dashed lines stretching from upper subplot to the lower connect the tail of the density profiles for cars and PTWs' traffic and the spacing between the two lines indicates the distance gap after PTWs overtake. . . . 27

15 Overtaking experiment density-space diagrams, upper subplot for PTWs and lower subplot for cars, free flow speed of $V_{2}=$ $1.8 \mathrm{~m} / \mathrm{s}$ greater than $V_{1}=1.5 \mathrm{~m} / \mathrm{s}$. . . . . . . . . . . 28

16 Speed vs. total number of vehicles plot, when free flow speed of PTWs less than cars and cars account to $80 \%$ of the total traffic, upper subplot Porous $G$, middle subplot Creeping, lower subplot $N$-pop. . . . . . . . . . . . . . . . . . . . . . . . 29

17 Flow-density diagram,for different penetration rates of PTWs. 31

18 Change in travel time of cars for different penetration rate of

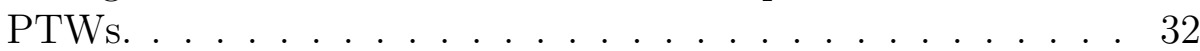

19 Evolution of number of vehicles in the queue over time. . . . . 33

20 Spatial distribution of the density of vehicles in the queue. . . 34 


\section{List of Tables}

1 Goodness of the fit measures obtained from the fitting experiments for different theoretical distributions. . . . . . . . . . . 11

2 Simulation Parameters . . . . . . . . . . . . . . . . 24

3 Speed values extracted at time $t=50 \mathrm{sec}$ and position $x=$ $39.15 m \ldots \ldots \ldots \ldots . \ldots \ldots$

4 The Change in Critical Density (veh/km per unit lane width) and Maximum Flow Rate (veh/hr/lane) at Different Ratios of PTWs . . . . . . . . . . . . . . . . 31

$5 \quad$ Table of Symbols And Acronym Used Along The Paper . . . . 43

6 The mean and variance of inter-vehicle spacing distribution for Poisson and uniform distribution assumptions. $\left[\rho_{1}, \rho_{2}\right]$ shows the traffic composition where $\rho_{1}$ and $\rho_{2}$ represent, respectively, PTWs and cars densities . . . . . . . . . . . . . . 44 
881 Table of symbols

\begin{tabular}{ll} 
Symbol & Meaning \\
\hline \hline$P T W$ & Powered Two Wheelers \\
$x$ & spatial location \\
$t$ & time \\
$q_{1} / 2$ & flow of PTWs/cars \\
$\rho_{1} / 2$ & density of PTWs $/$ cars \\
$v_{1} / 2$ & speed of PTWs $/$ cars \\
$v_{1} /{ }^{f}$ & free flow speed of PTWs $/$ cars \\
$i$ & vehicle class \\
$R$ & radius of circle \\
$l_{p}$ & length of Delaunay edge for points \\
$l_{c}$ & length of Delaunay edge for circles \\
\hline
\end{tabular}

Table 5: Table of Symbols And Acronym Used Along The Paper 

process for the spatial distribution of vehicles.

\begin{tabular}{l|ccccc}
{$\left[\rho_{1}, \rho_{2}\right]$} & {$[0.005,0.005]$} & {$[0.02,0.01]$} & {$[0.05,0.02]$} & {$[0.1,0.05]$} & {$[0.15,0.075]$} \\
Mean & 16.57 & 7.84 & 4.39 & 2.15 & 1.471 \\
Variance & 234.5 & 72.89 & 26.70 & 5.677 & 2.02 \\
\hline \multirow{7}{*}{ Mean } & 17.04 & 8.24 & 4.415 & 2.15 & 1.41 \\
Variance & 223.5 & 76.45 & 26.23 & 5.75 & 1.95
\end{tabular}

Table 6: The mean and variance of inter-vehicle spacing distribution for Poisson and uniform distribution assumptions. $\left[\rho_{1}, \rho_{2}\right]$ shows the traffic composition where $\rho_{1}$ and $\rho_{2}$ represent, respectively, PTWs and cars densities 